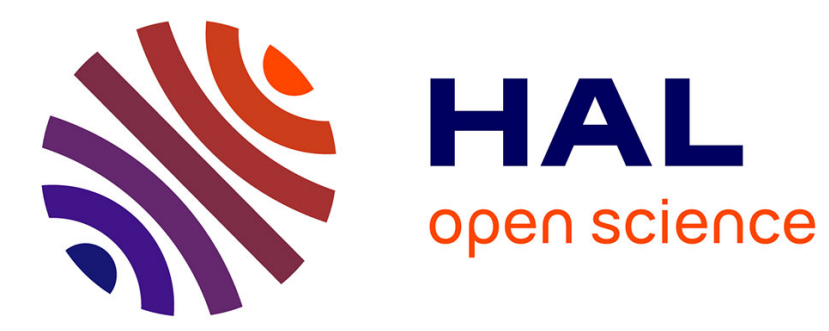

\title{
Multiscale analysis of defective multiple-Hopf bifurcations
}

\author{
Angelo Luongo, Angelo Di Egidio, Achille Paolone
}

\section{To cite this version:}

Angelo Luongo, Angelo Di Egidio, Achille Paolone. Multiscale analysis of defective multiple-Hopf bifurcations. Computers \& Structures, 2004, 82 (31-32), pp.2705-2722. hal-00794677

\section{HAL Id: hal-00794677 \\ https://hal.science/hal-00794677}

Submitted on 26 Feb 2013

HAL is a multi-disciplinary open access archive for the deposit and dissemination of scientific research documents, whether they are published or not. The documents may come from teaching and research institutions in France or abroad, or from public or private research centers.
L'archive ouverte pluridisciplinaire HAL, est destinée au dépôt et à la diffusion de documents scientifiques de niveau recherche, publiés ou non, émanant des établissements d'enseignement et de recherche français ou étrangers, des laboratoires publics ou privés. 


\title{
Multiscale analysis of defective multiple-Hopf bifurcations
}

\author{
Angelo Luongo ${ }^{a, *}$, Angelo Di Egidio ${ }^{a, 1}$, Achille Paolone ${ }^{b, 2}$ \\ a Dipartimento di Ingegneria delle Strutture delle Acque e del Terreno, Università di L'Aquila, Piazzale E Pontieri 2, \\ 67040 Monteluco di Roio, L'Aquila, Italy \\ b Dipartimento di Ingegneria delle Strutture e Geotecnica, Università di Roma "La Sapienza", via Eudossiana 18, 00184 Rome, Italy
}

\begin{abstract}
An adapted version of the Multiple Scale Method is formulated to analyze 1:1 resonant multiple Hopf bifurcations of discrete autonomous dynamical systems, in which, for quasi static variations of the parameters, an arbitrary number $m$ of critical eigenvalues simultaneously crosses the imaginary axis. The algorithm therefore requires discretizing con tinuous systems in advance. The method employs fractional power expansion of a perturbation parameter, both in the state variables and in time, as suggested by a formal analogy with the eigenvalue sensitivity analysis of nilpotent (defec tive) matrices, also illustrated in detail. The procedure leads to an order $m$ differential bifurcation equation in the com plex amplitude of the unique critical eigenvector, which is able to capture the dynamics of the system around the bifurcation point. The procedure is then adapted to the specific case of a double Hopf bifurcation $(m \quad 2)$, for which a step by step, computationally oriented version of the method is furnished that is directly applicable to solve practical problems. To illustrate the algorithm, a family of mechanical systems, subjected to aerodynamic forces triggering 1:1 resonant double Hopf bifurcations is considered. By analyzing the relevant bifurcation equation, the whole scenario is described in a three dimensional parameter space, displaying rich dynamics.
\end{abstract}

Keywords: Resonant Hopf bifurcations; Multiple scale method; Coalescent eigenvalues; Nilpotent matrices; Fractional powers expansions; Homoclinic bifurcations; Quasi periodic motions

\section{Introduction}

Autonomous dynamic systems subjected to quasi sta tic variations in their parameters usually modify their

\footnotetext{
* Corresponding author. Tel.: +39 0862 434511/434521; fax: +390862434548.

E mail addresses: luongo@ing.univaq.it (A. Luongo), ade@ing.univaq.it (A.D. Egidio), achille.paolone@uniroma1.it (A. Paolone).

1 Tel.: +390862 434530 .

2 Tel.: +3906 44585193 .
}

equilibrium state. Small variations around a stable equi librium point do not modify the qualitative nature of the local flow in the state space, so that the dynamics remain substantially unaltered. However, for sufficiently large variations of the parameters, a sudden qualitative mod ification of the flow can take place, typically entailing a lose of stability of the equilibrium state. In this case a bifurcation is said to occur. Bifurcations from an equilib rium point are governed by the eigenvalues of the Jaco bian matrix at the point. A simple bifurcation occurs when one of the eigenvalues first crosses the imaginary axis on the complex plane. The bifurcation is said to 
be static (or divergence) if the critical eigenvalue is zero, while it is called dynamic (or Hopf) if it has a non zero imaginary part. Simple bifurcations are structurally sta ble in families of one parameter systems (i.e. have the same properties of sufficiently close systems or, in other words, are robust under small perturbations [1]). A mul tiple bifurcation manifests itself when a cluster of eigen values is simultaneously critical. It is structurally stable in families of multi parameter systems. The objective of the bifurcation analysis is to study the system dynam ics around the bifurcation point in the parameter space.

The most popular method used to investigate bifur cations is the center manifold method (CMM) [1,2]. This consists (a) in finding the manifold of the state space to which the dynamics asymptotically tend, and (b) in reducing the multi (or even infinite) dimensional sys tem to an equivalent low dimensional system, which de scribes the essential dynamics that develop on the manifold. In recent years the authors have developed an alternative approach, based on the multiple scale method (MSM) [3], which furnishes the reduced equation of the motion without describing the center manifold in advance [4 7]. The main results of the analysis are re sumed in a review paper [8].

Attention is here focused on multiple Hopf bifurca tions. In [9], nonresonant double Hopf bifurcations (which occur when the imaginary parts of the critical eigen values are in an irrational ratio) were studied using the CMM. More recently, the CMM has been employed to analyze a vibro impact system that exhibits the same type of double bifurcation [10]. In [4] nonresonant dou ble Hopf bifurcations are studied by the MSM. Succes sively, the MSM is applied in [11] to analyze simple Hopf bifurcations occurring in delay systems and in [12] to study nonresonant double Hopf bifurcations, where an automatic procedure to analyze the critical sce nario is developed. In $[5,13,14]$ resonant double Hopf bifurcations of types 1:2 and 1:3 were analyzed using the MSM. The more difficult case of 1:1 resonance, in which the two couples of imaginary eigenvalues coa lesce, was addressed in [15], by applying the CMM, and in [22] by the MSM.

In this paper an algorithm based on the MSM is implemented to study 1:1 resonant $m$ Hopf bifurcations, in which $m$ imaginary eigenvalues coalesce at the critical point, with $m$ being arbitrary. The method is then spe cialized to the case in which $m \quad 2$ and a computation ally oriented version is furnished. The algorithm applies to finite dimensional discrete systems. Therefore, if the system under study is infinite dimensional continu ous) it must be discretized in advance, through a finite element or Galerkin approach, before the procedure is applied. Other reduction methods, such as those de scribed in [18], which account for the passive (i.e. non critical modes), are not strictly necessary if a numerical approach is adopted, since the MSM itself selects the passive contributions, provided the discretization proc ess performed is sufficiently refined. Direct applications of the MSM to continuous systems will constitute the object of forthcoming papers [17].

\section{Problem position}

An $N$ dimensional, autonomous, dynamical system is considered, of state variables $\mathbf{x} \in \mathrm{R}^{N}$, depending on a set $\boldsymbol{\mu} \in R^{M}$ of control parameters. Let

$\dot{\mathbf{x}}=\mathbf{F}(\mathbf{x}, \boldsymbol{\mu})$

be the equation of motion governing the free evolution of the system. It is assumed that Eq. (1) admits the triv ial equilibrium path $\mathbf{x} \quad \mathbf{0}, \forall \boldsymbol{\mu}$, i.e. it has previously been reduced by a suitable change of variable to the so called local form. It is further admitted that $(\mathbf{x}, \boldsymbol{\mu}) \quad(\mathbf{0 , 0}, \mathbf{0})$ is a bifurcation point $O$, at which the Jacobian matrix $\mathbf{F}_{\mathbf{x}}^{0}:=\mathbf{F}_{\mathbf{x}}(0,0)$ admits a couple of eigenvalues $\lambda_{0} \quad \pm \mathrm{i} \omega$ having algebraic multiplicity $m>1$, the remaining eigen values being all stable. The codimension of the manifold in the parameter space on which such a bifurcation oc curs (here referred to as the linear codimension of the bifurcation [8]) is equal to $M:=2 m \quad 1$, since the van ishing of the real part of the eigenvalues requires that $m$ conditions be satisfied, while the coalescence of their imaginary parts entails $m \quad 1$ additional conditions. In order to render the bifurcation structurally stable, an $M$ parameter family of dynamical systems (Eq. (1)) is therefore considered, in which the critical system natu rally appears at point $O$.

Except for special cases, of higher codimension, only one critical eigenvector $\mathbf{u}$ exists associated with $\lambda_{0}$, so that the matrix $\mathbf{F}_{\mathbf{x}}^{0}$ has an incomplete set of eigenvectors (and for this reason is often called a defective or nilpotent matrix). It is well known from algebra that a chain of $m$ generalized (right) eigenvectors can be built up to com plete the base, by recursively solving the equations:

$\left(\mathbf{F}_{\mathbf{x}}^{0} \quad \lambda_{0} \mathbf{I}\right) \mathbf{u}_{k}=\mathbf{u}_{k-1} \quad k=2,3, \ldots, m$

where $\mathbf{u}_{1} \equiv \mathbf{u}$ is the proper right eigenvector. A complete base of left generalized eigenvectors can also be found by recursively solving the equations:

$\left(\begin{array}{ll}\mathbf{F}_{\mathbf{x}}^{0} & \lambda_{0} \mathbf{I}\end{array}\right)^{\mathrm{H}} \mathbf{v}_{j-1}=\mathbf{v}_{j} \quad j=m, m \quad 1, \ldots, 2$

where $\mathrm{H}$ denotes the transposed conjugate and $\mathbf{v}_{m}$ is the (unique) proper left eigenvector. The left and right eigenvectors satisfy the orthonormalization properties $\mathbf{v}_{j}^{\mathrm{H}} \mathbf{u}_{k}=\delta_{j k}$. This means that all the eigenvectors $\mathbf{u}_{k}$ of the chain, except for the higher order eigenvector $\mathbf{u}_{m}$, be long to the range of the operator $\mathbf{F}_{\mathbf{x}}^{0} \quad \lambda_{0} \mathbf{I}$. This circum stance differs from that occurring in the non defective case $(m \quad 1)$ for which $\mathbf{v}_{1}^{\mathrm{H}} \mathbf{u}_{1} \neq 0$.

The aim of the analysis is to investigate the dynamics of the nonlinear system around the bifurcation point $O$. 


\section{Multiple-Hopf bifurcation analysis}

The multiscale perturbation method [3] is applied to analyze defective codimension $M$ Hopf bifurcations. The method calls for the following steps, which will be described in detail ahead: (a) expand Eq. (1) in a Mc Laurin series of $(\mathbf{x}, \boldsymbol{\mu})$, namely ${ }^{3}$

$$
\begin{aligned}
\dot{\mathbf{x}}= & \mathbf{F}_{\mathbf{x}}^{0} \mathbf{x}+\frac{1}{2} \mathbf{F}_{\mathbf{x x}}^{0} \mathbf{x}^{2}+\mathbf{F}_{\mathbf{x} \boldsymbol{x}}^{0} \mathbf{x} \boldsymbol{\mu}+\frac{1}{6} \mathbf{F}_{\mathbf{x x x}}^{0} \mathbf{x}^{3}+\frac{1}{2} \mathbf{F}_{\mathbf{x x} \boldsymbol{\mu}}^{0} \mathbf{x}^{2} \boldsymbol{\mu} \\
& +\frac{1}{2} \mathbf{F}_{\mathbf{x} \mu \mu}^{0} \mathbf{x} \boldsymbol{\mu}^{2}+\cdots
\end{aligned}
$$

where an index denotes differentiation of the vector $\mathbf{F}$ with respect to $\mathbf{x}$ or $\boldsymbol{\mu}$; (b) expand the state vector $\mathbf{x}$ in a suitable series of a perturbation parameter $\varepsilon$; then scale the parameter vector $\boldsymbol{\mu}$ by some suitable power of $\varepsilon$, i.e. $\boldsymbol{\mu}=\varepsilon^{\alpha} \hat{\boldsymbol{\mu}}$; then introduce several independent time scales $t_{k}=\varepsilon^{\beta_{k}} t$ for some $\beta_{k}$ 's; (c) build up the perturbation equations by separately equating the terms of the same power of $\varepsilon$ to zero in Eq. (4); (d) solve these equations in sequence and enforce solvability (Freedholm) condi tions at each step, obtaining amplitude modulation equa tions on different time scales $t_{k}$; (e) recombine the amplitude equations by returning to the true time $t$ (reconstitution procedure $[19,20]$ ), in order to draw the so called bifurcation equation; (f) solve (often numeri cally) this nonlinear equation to determine the nature of the solution in different regions of the parameter space around point $O$ (i.e. build up the stability diagram).

All the steps, except for (b), are the same as those performed in standard analysis of non defective bifurca tions. However, while in that analysis integer powers of $\varepsilon$ are employed, here fractional powers must be used both in the state variable expansions and in the slow time scales. Indeed, if one attempts to address the problem by the standard method, the procedure will fail, due to the fact that $\mathbf{v}_{m}^{\mathrm{H}} \mathbf{u}_{1}=0$ [8]. This circumstance is similar to that which occurs in sensitivity analysis of defective eigenvalues [21]. The order of the powers to be used, however, is not known a priori, and this represents the major difficulty in formulating the algorithm. By exploit ing the analogy with the sensitivity of non singular pert urbations, powers of order $\varepsilon^{1 / m}$ were successfully applied

${ }^{3}$ Here the notation Guv, Guvw,..., is used to denote multilinear forms of degree $2,3, \ldots$, i.e.:

Guv $\sum_{i}^{N} \sum_{j}^{N} g_{i j} u_{i} u_{j}, \quad$ Guvw $\sum_{i=1}^{N} \sum_{j}^{N} \sum_{k=1}^{N} g_{i j k} u_{i} u_{j} u_{k}$

Therefore:

$\left.\mathbf{F}_{\mathbf{x} \mathbf{x}}^{0} \mathbf{x}^{2} \sum_{i}^{N} \sum_{j}^{N} \frac{\partial \mathbf{F}}{\partial x_{i} \partial x_{j}}\right|_{\mathbf{0}} x_{i} x_{j}$

and similar expressions hold in Eq. (4). in [7] to analyze multiple zero bifurcations of any codi mension. Nevertheless, it is easy to check that, although that algorithm also works for defective multiple Hopf bifurcations, it is not efficient, since it entails trivial steps in which several quantities turn out to be identically zero. Moreover, such steps depend on the algebraic mul tiplicity $m$ of the critical eigenvalue being even or odd. An alternative efficient procedure is developed here, again suggested by the sensitivity of defective eigen values to singular perturbations. In Appendix A the analogy is detailed, and the motivation for the choice of the fractional powers to be used is given. The two cases, in which $m$ is either even or odd, are dealt with separately.

\subsection{Even $m$ case}

The following series expansion for $\mathbf{x}$ and scaling for $\boldsymbol{\mu}$ are adopted:

$\boldsymbol{\mu}=\varepsilon^{2} \hat{\boldsymbol{\mu}}$

$\mathbf{x}=\varepsilon\left(\mathbf{x}_{0}+\varepsilon^{2 / m} \mathbf{x}_{2}+\varepsilon^{4 / m} \mathbf{x}_{4}+\cdots\right)$

i.e. odd powers of $\varepsilon^{1 / m}$ are omitted in the expansion of $\mathbf{x} /$ $\varepsilon$. The state variables $\mathbf{x}$ are assumed to depend on several time scales $t_{i}$, for which $\mathbf{x}_{k} x_{k}\left(t_{i}\right)$. By again omitting odd terms and letting

$t_{0}=t, \quad t_{2}=\varepsilon^{2 / m} t, \quad t_{4}=\varepsilon^{4 / m} t, \ldots$

the chain rule furnishes

$\mathrm{d} / \mathrm{d} t=d_{0}+\varepsilon^{2 / m} d_{2}+\varepsilon^{4 / m} d_{4}+\cdots$

where $d_{k}:=\partial / \partial t_{k}(k \quad 2,4, \ldots)$. Substitution of Eqs. (5) and (7) in Eqs. (4), leads to the following perturbation equations, written up to the $\varepsilon^{3+2 / m}$ order

$$
\begin{aligned}
& \varepsilon: \quad\left(\begin{array}{ll}
d_{0} & \mathbf{F}_{\mathbf{x}}^{0}
\end{array}\right) \mathbf{x}_{0}=0
\end{aligned}
$$

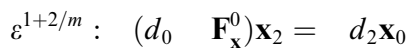

$$
\begin{aligned}
& \varepsilon^{1+4 / m}:\left(\begin{array}{ll}
d_{0} & \mathbf{F}_{\mathbf{x}}^{0}
\end{array}\right) \mathbf{x}_{4}=d_{2} \mathbf{x}_{2} \quad d_{4} \mathbf{x}_{0}
\end{aligned}
$$

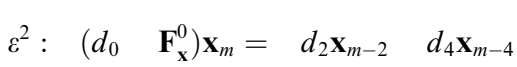

$$
\begin{aligned}
& +\cdots+1 / 2 \mathbf{F}_{\mathbf{x x}}^{0} \mathbf{x}_{0}^{2}
\end{aligned}
$$

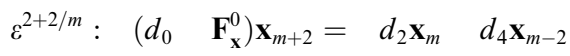

$$
\begin{aligned}
& +\cdots+\mathbf{F}_{\mathbf{x x}}^{0} \mathbf{x}_{0} \mathbf{x}_{2} \\
& \varepsilon^{3}: \quad\left(\begin{array}{lll}
d_{0} & \mathbf{F}_{\mathbf{x}}^{0}
\end{array}\right) \mathbf{x}_{2 m}=d_{2} \mathbf{x}_{2 m-2} \quad d_{4} \mathbf{x}_{2 m-4} \\
& +\cdots+\mathbf{F}_{\mathbf{x x}}^{0}\left(\mathbf{x}_{0} \mathbf{x}_{m}+\mathbf{x}_{2} \mathbf{x}_{m-2}+\cdots\right) \\
& +1 / 6 \mathbf{F}_{\mathbf{x x x}}^{0} \mathbf{x}_{0}^{3}+\mathbf{F}_{\mathbf{x} \boldsymbol{\mu}}^{0} \mathbf{x}_{0} \hat{\boldsymbol{\mu}} \\
& \varepsilon^{3+2 / m}:\left(\begin{array}{lll}
d_{0} & \mathbf{F}_{\mathbf{x}}^{0}
\end{array}\right) \mathbf{x}_{2 m+2}=d_{2} \mathbf{x}_{2 m} \quad d_{4} \mathbf{x}_{2 m-2}+\cdots \\
& +\mathbf{F}_{\mathbf{x x}}^{0}\left(\mathbf{x}_{0} \mathbf{x}_{m+2}+\mathbf{x}_{2} \mathbf{x}_{m}+\cdots\right) \\
& +1 / 2 \mathbf{F}_{\mathbf{x x x}}^{0} \mathbf{x}_{0}^{2} \mathbf{x}_{2}+\mathbf{F}_{\mathbf{x} \boldsymbol{\mu}}^{0} \mathbf{x}_{2} \hat{\boldsymbol{\mu}}
\end{aligned}
$$


By solving these equations in sequence and using Eqs. (2) with $\lambda_{0} i \omega$, the following solutions are drawn:

$$
\begin{aligned}
\varepsilon: & \mathbf{x}_{0}=A\left(t_{1}, t_{2}, \ldots\right) \mathbf{u}_{1} \mathrm{e}^{\mathrm{i} \omega t_{0}} \\
\varepsilon^{1+2 / m}: & \mathbf{x}_{2}=d_{2} A \mathbf{u}_{2} \mathrm{e}^{\mathrm{i} \omega t_{0}} \\
\varepsilon^{1+4 / m}: & \mathbf{x}_{4}=\left(d_{2}^{2} A \mathbf{u}_{3}+d_{4} A \mathbf{u}_{2}\right) \mathrm{e}^{\mathrm{i} \omega t_{0}}
\end{aligned}
$$

in which $A$ is a complex amplitude depending in an un known way on the slow time scales. In Eqs. (9) the homogeneous solutions are neglected at higher levels since they repeat the lowest order solution. If they were accounted for, additional arbitrary amplitudes would appear that, however, could be reabsorbed in the unique unknown $A$ at the end of the procedure. It should be noted that Eqs. (8) can be solved up to the $\varepsilon^{3}$ order with out requiring solvability, since all the resonant terms, being linear combinations of the first $m \quad 1$ generalized eigenvectors, belong to the range of the operator. This is a peculiar aspect of defective systems, not occurring in generic systems, as discussed in detail in $[8,21]$. In partic ular, $\mathbf{x}_{2}, \mathbf{x}_{4}, \ldots, \mathbf{x}_{m-2}$ only contain the simple harmonic $\omega$, while $\mathbf{x}_{m}, \mathbf{x}_{m+2}, \ldots, \mathbf{x}_{2 m-2}$ also contain the double har monic $2 \omega$, in addition to the constant term generated by the quadratic nonlinearities. When the $\varepsilon^{3}$ order equation is reached, the resonant terms finally appear, produced by the cubic nonlinearities $\mathbf{F}_{\mathbf{x x x}}^{0} \mathbf{x}_{0}^{3}$, the quadratic nonline arities $\mathbf{F}_{\mathbf{x x}}^{0} \mathbf{x}_{0} \mathbf{x}_{m}$, and the parameter dependent term $\mathbf{F}_{\mathrm{x} \mu}^{0} \mathbf{x}_{0} \hat{\boldsymbol{\mu}}$. These resonant terms, which are proportional to $A^{2} A$ and $A \hat{\boldsymbol{\mu}}$, all enter the first solvability condition, which therefore has the following structure:

$\varepsilon^{3}: \quad d_{2}^{m} A=\mathscr{L}_{m}\left(A \hat{\boldsymbol{\mu}}, A^{2} A\right)$

where $\mathscr{L}$ is an algebraic linear operator. This is a nonlin ear differential equation of the $m$ th order governing the amplitude evolution on the $t_{2}$ scale (amplitude modula tion equation). In order to account for slower modula tions, one has to proceed to the higher orders. There, solvability conditions furnish amplitude equations of the first order in the derivative of the highest scales, of the type:

$\varepsilon^{3+2 / m}: \quad m d_{4} d_{2}^{m-1} A=\mathscr{L}_{m+1}\left(\hat{\boldsymbol{\mu}} d_{2} A, A A d_{2} A, A^{2} d_{2} A\right)$

By observing that, by virtue of Eq. (7), $\mathrm{d}^{m} A / \mathrm{d} t^{m}=$ $\left(\varepsilon^{2 / m} d_{2}+\varepsilon^{4 / m} d_{4}+\ldots\right)^{m} A=\varepsilon\left(\varepsilon^{2} d_{2}^{m}+\varepsilon^{2+2 / m} m d_{4} d_{2}^{m-1}+\cdots\right) A$, all the solvability conditions can be combined to obtain an unique differential equation of order $m$, namely

$\frac{\mathrm{d}^{m} A}{\mathrm{~d} t^{m}}=\mathscr{L}\left(\boldsymbol{\mu} A, A^{2} A ; \boldsymbol{\mu} \dot{A}, A A \dot{A}, A^{2} \dot{A} ; \ldots\right)$

to be referred to as the reconstituted modulation equation or, more simply, as the bifurcation equation. In it, the parameter $\varepsilon$ has been reabsorbed according to the rules $\varepsilon A \rightarrow A, \varepsilon^{2} \hat{\boldsymbol{\mu}} \rightarrow \boldsymbol{\mu}$, and $\varepsilon^{2 / m} \mathrm{~d} / \mathrm{d} t \rightarrow \mathrm{d} / \mathrm{d} t$. In Eq. (12) $d^{m} A / \mathrm{d} t^{m}$ is a term of the $\varepsilon^{3}$ order, while the right hand member contains (separated by semicolons) all the terms associated with frequency $\omega$ of the order $\varepsilon^{3}, \varepsilon^{3+2 / m}, \ldots$, up to the highest order accounted for in the analysis For example, if $m \quad 4$, the bifurcation equation at the $\varepsilon^{4}$ order reads:

$$
\dddot{A}=\mathscr{L}\left(\boldsymbol{\mu} A, A^{2} A ; \boldsymbol{\mu} \dot{A}, A A \dot{A}, A^{2} \dot{A} ; \boldsymbol{\mu} \ddot{A}, \dot{A}^{2} A, A \dot{A} \dot{A}\right)
$$

\subsection{Odd $m$ case}

If $m$ is odd, a complete series is adopted for $\mathbf{x}$, namely $\mathbf{x}=\varepsilon\left(\mathbf{x}_{0}+\varepsilon^{1 / m} \mathbf{x}_{1}+\varepsilon^{2 / m} \mathbf{x}_{2}+\cdots\right)$

while the same ordering (Eq. $\left.\left(5_{2}\right)\right)$ for $\boldsymbol{\mu}$ and the same (even) time scales (Eq. (6)) are maintained, as in the even $m$ case. The following, more involved, perturbation equations are drawn

$$
\begin{aligned}
& \varepsilon: \quad\left(\begin{array}{ll}
d_{0} & \left.\mathbf{F}_{\mathbf{x}}^{0}\right) \mathbf{x}_{0}=0
\end{array}\right. \\
& \varepsilon^{1+1 / m}: \quad\left(d_{0} \quad \mathbf{F}_{\mathbf{x}}^{0}\right) \mathbf{x}_{1}=0
\end{aligned}
$$

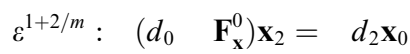

$$
\begin{aligned}
& \varepsilon^{1+3 / m}: \quad\left(d_{0} \quad \mathbf{F}_{\mathbf{x}}^{0}\right) \mathbf{x}_{3}=d_{2} \mathbf{x}_{1} \\
& \varepsilon^{1+4 / m}:\left(\begin{array}{ll}
d_{0} & \mathbf{F}_{\mathbf{x}}^{0}
\end{array}\right) \mathbf{x}_{4}=d_{2} \mathbf{x}_{2} \quad d_{4} \mathbf{x}_{0} \\
& \varepsilon^{2}: \quad\left(\begin{array}{ll}
d_{0} & \mathbf{F}_{\mathbf{x}}^{0}
\end{array}\right) \mathbf{x}_{m}=d_{2} \mathbf{x}_{m-2} \quad d_{4} \mathbf{x}_{m-4} \\
& +\cdots+1 / 2 \mathbf{F}_{\mathbf{x x}}^{0} \mathbf{x}_{0}^{2}
\end{aligned}
$$

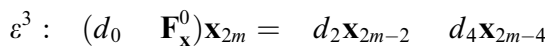

$$
\begin{aligned}
& +\cdots+\mathbf{F}_{\mathbf{x x}}^{0}\left(\mathbf{x}_{0} \mathbf{x}_{m}+\mathbf{x}_{1} \mathbf{x}_{m-1}+\cdots\right) \\
& +1 / 6 \mathbf{F}_{\mathbf{x x x}}^{0} \mathbf{x}_{0}^{3}+\mathbf{F}_{\mathbf{x \mu}}^{0} \mathbf{x}_{0} \hat{\boldsymbol{\mu}} \\
& \varepsilon^{3+1 / m}: \quad\left(\begin{array}{ll}
d_{0} & \left.\mathbf{F}_{\mathbf{x}}^{0}\right) \mathbf{x}_{2 m+1}=d_{2} \mathbf{x}_{2 m-1} \quad d_{4} \mathbf{x}_{2 m-3}
\end{array}\right. \\
& +\cdots+\mathbf{F}_{\mathbf{x x}}^{0}\left(\mathbf{x}_{0} \mathbf{x}_{m+1}+\mathbf{x}_{1} \mathbf{x}_{m}+\cdots\right) \\
& +1 / 2 \mathbf{F}_{\mathbf{x x x}}^{0} \mathbf{x}_{0}^{2} \mathbf{x}_{1}+\mathbf{F}_{\mathbf{x} \boldsymbol{\mu}}^{0} \mathbf{x}_{1} \hat{\boldsymbol{\mu}}
\end{aligned}
$$

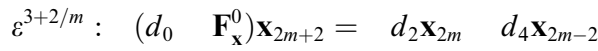

$$
\begin{aligned}
& +\cdots+\mathbf{F}_{\mathbf{x x}}^{0}\left(\mathbf{x}_{0} \mathbf{x}_{m+2}+\mathbf{x}_{1} \mathbf{x}_{m+1}+\cdots\right) \\
& +1 / 2 \mathbf{F}_{\mathbf{x x x}}^{0} \mathbf{x}_{0}^{2} \mathbf{x}_{2}+\mathbf{F}_{\mathbf{x} \boldsymbol{\mu}}^{0} \mathbf{x}_{2} \hat{\boldsymbol{\mu}} \\
& +1 / 2 \mathbf{F}_{\mathbf{x x x}}^{0} \mathbf{x}_{0} \mathbf{x}_{1}^{2}
\end{aligned}
$$

Since $\mathbf{x}_{0}$ is still given by Eq. $\left(9_{1}\right), \mathbf{x}_{2}, x_{4}, \ldots, \mathbf{x}_{2 m-2}$ re main unchanged in relation to the previous case (see Eqs. (9)); in particular they only contain the fundamen tal harmonic. Moreover, since $\mathbf{x}_{1} 0$, then $\mathbf{x}_{3}$, $\mathbf{x}_{5}, \ldots, \mathbf{x}_{m-2}$ also vanish, while $\mathbf{x}_{m}, x_{m+2}, \ldots, \mathbf{x}_{2 m+1}$ are sums of double and zero harmonics. Therefore the even terms of the series $\mathbf{x}_{0}, x_{2}, \ldots, \mathbf{x}_{2 m}, \mathbf{x}_{2 m+2}, \ldots$, are identical to those of Eqs. (8). When the $\varepsilon^{3}$ perturbation equation is considered, resonant terms appear through the mech anism illustrated above, leading to a solvability condi 
tion identical to Eq. (10). At the subsequent orders no resonant terms appear in the equations in the odd terms. Indeed, quadratic nonlinearities involve products $\mathbf{x}_{i} \mathbf{x}_{j}$, in which $(i, j)$ are either even or odd and therefore of even resultant frequencies; similarly, cubic nonlinearities involve products $\mathbf{x}_{i} \mathbf{x}_{j} \mathbf{x}_{k}$ in which $(i, j, k)$ are either all odd or two even and one odd and therefore still of even fre quencies. Solvability is thus required only for the equations in the even terms $\mathbf{x}_{2 k}$, which are still of type (11). Reconstituted bifurcation equations in the form (12) are thus obtained, both for even and odd multiplic ity $m$.

\section{Double Hopf-bifurcation}

The multiple scale procedure developed in the previ ous section is specialized to a system exhibiting a double imaginary eigenvalue. By assuming $m \quad 2$ in the series expansion $(5)_{2}$, this contains, as a particular case, integer powers of $\varepsilon$ only. The relevant perturbation equations (8) become

$$
\begin{aligned}
& \varepsilon: \quad\left(\begin{array}{ll}
d_{0} & \mathbf{F}_{\mathbf{x}}^{0}
\end{array}\right) \mathbf{x}_{0}=\mathbf{0}
\end{aligned}
$$

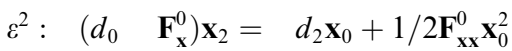

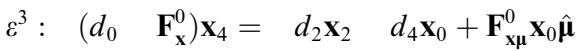

$$
\begin{aligned}
& +\mathbf{F}_{\mathbf{x x}}^{0} \mathbf{x}_{0} \mathbf{x}_{2}+1 / 6 \mathbf{F}_{\mathbf{x x x}}^{0} \mathbf{x}_{0}^{3} \\
& \varepsilon^{4}: \quad\left(\begin{array}{lllll}
d_{0} & \mathbf{F}_{\mathbf{x}}^{0}
\end{array}\right) \mathbf{x}_{6}=d_{2} \mathbf{x}_{4} \quad d_{4} \mathbf{x}_{2} \quad d_{6} \mathbf{x}_{0} \\
& +\mathbf{F}_{\mathbf{x} \boldsymbol{\mu}}^{0} \mathbf{x}_{2} \hat{\boldsymbol{\mu}}+\mathbf{F}_{\mathbf{x x}}^{0}\left(\mathbf{x}_{0} \mathbf{x}_{4}+\mathbf{x}_{2}^{2}\right) \\
& +\mathbf{F}_{\mathbf{x x \mu}}^{0} \mathbf{x}_{0}^{2} \hat{\boldsymbol{\mu}}+1 / 2 \mathbf{F}_{\mathbf{x x x}}^{0} \mathbf{x}_{0}^{2} \mathbf{x}_{2}
\end{aligned}
$$

By following the steps previously illustrated, the follow ing solutions are found:

$$
\begin{aligned}
\mathbf{x}_{0}= & A\left(t_{1}, t_{2}, \ldots\right) \mathbf{u}_{1} \mathrm{e}^{\mathrm{i} \omega t_{0}}+\text { c.c. } \\
\mathbf{x}_{2}= & d_{2} A \mathbf{u}_{2} \mathrm{e}^{\mathrm{i} \omega t_{0}}+1 / 2 A^{2} \mathbf{z}_{11} \mathrm{e}^{\mathrm{i} 2 \omega t_{0}}+1 / 2 A A \mathbf{z}_{11}+\text { c.c. } \\
\mathbf{x}_{4}= & \left(d_{4} A \mathbf{u}_{2}+A^{2} A \mathbf{z}_{111}+A \mathbf{Z}_{1 \mu} \hat{\boldsymbol{\mu}}\right) \mathrm{e}^{\mathrm{i} \omega_{0} t_{0}} \\
& +A d_{2} A \mathbf{z}_{12} \mathrm{e}^{\mathrm{i} 2 \omega_{0} t_{0}} \\
& +A^{3} \mathbf{z}_{111} \mathrm{e}^{\mathrm{i} 3 \omega_{0} t_{0}}+A d_{2} A+\mathbf{z}_{12}+\text { c.c. }
\end{aligned}
$$

where $\mathbf{x}_{0}$ is the generating solution and $\mathbf{z}_{i j} \in Z^{N}$, $\mathbf{Z}_{1 \boldsymbol{\mu}} \in \mathrm{Z}^{N} \times \mathrm{Z}^{M}$ are solutions of linear algebraic equa tions reported in Appendix B.

Similarly, the following solvability conditions are found at the various orders:

$$
\begin{array}{ll}
\varepsilon^{3}: & d_{2}^{2} A=\mathbf{C}_{1 \mu} \widehat{\boldsymbol{\mu}} A+C_{111} A^{2} A \\
\varepsilon^{4}: & 2 d_{2} d_{4} A=\mathbf{C}_{2 \mu} \widehat{\boldsymbol{\mu}} d_{2} A+C_{112} A A d_{2} A+C_{112} A^{2} d_{2} A
\end{array}
$$

where the coefficients $C_{i j k} \in \mathrm{Z}$ and $\mathbf{C}_{j \boldsymbol{\mu}} \in \mathrm{Z} \times \mathrm{Z}^{M}$ are also defined in Appendix B. By coming back to the true time $t$, Eqs. (18) are combined in a single equation. By taking into account that:

$$
\frac{\mathrm{d}^{2} A}{\mathrm{~d} t^{2}}=\varepsilon^{3}\left(d_{2}^{2}+2 \varepsilon d_{2} d_{4}\right) A+O\left(\varepsilon^{5}\right)
$$

Eqs. (18) lead to the following bifurcation equation:

$$
\begin{aligned}
\frac{\mathrm{d}^{2} A}{\mathrm{~d} t^{2}}= & \mathbf{C}_{1 \mu} \boldsymbol{\mu} A+\mathbf{C}_{2 \boldsymbol{\mu}} \boldsymbol{\mu} \frac{\mathrm{d} A}{\mathrm{~d} t}+C_{111} A^{2} A+C_{112} A A \frac{\mathrm{d} A}{\mathrm{~d} t} \\
& +C_{112} A^{2} \frac{\mathrm{d} A}{\mathrm{~d} t}
\end{aligned}
$$

where the parameter $\varepsilon$ has been reabsorbed according the rules: $\varepsilon A \rightarrow A, \varepsilon \mathrm{d} / \mathrm{d} t \rightarrow \mathrm{d} / \mathrm{d} t, \varepsilon^{2} \widehat{\boldsymbol{\mu}} \rightarrow \boldsymbol{\mu}$. Eq. (20) gov erns the system's asymptotic dynamics (i.e. for a suffi ciently large time, for which the contribution of stable eigenvalues has been extinguished). It is equivalent to two first order equations in the two complex variables $\left(A_{1}, A_{2}\right):=(A, \dot{A})$.

To express the bifurcation equations in real form, it is convenient to adopt a mixed (polar and Cartesian) form for the complex amplitudes [22]

$A_{1}=\frac{1}{2} a \mathrm{e}^{\mathrm{i} \theta}, \quad A_{2}=\frac{1}{2}(u+\mathrm{i} v) \mathrm{e}^{\mathrm{i} \theta}$

Substituting Eqs. (21) into (20) and separating real and imaginary parts yields:

$$
\left\{\begin{array}{l}
\dot{a}=u \\
\dot{u}=g(a, u, v, \dot{\theta} ; \boldsymbol{\mu}) \\
\dot{v}=h(a, u, v, \dot{\theta} ; \boldsymbol{\mu}) \\
a \dot{\theta}=v
\end{array}\right.
$$

where $g$ and $h$ are cubic polynomials in the variables $(a, u, v)$. From Eq. $\left(22_{4}\right), \dot{\theta}$ can be expressed as a function of $a$ and $v$ and then substituted in $\left(22_{2}\right)$ and $\left(22_{3}\right)$. It fol lows that the amplitude modulation equations $\left(22_{1,2,3}\right)$ (equal in number to the linear codimension of the prob lem $M \quad 3$ ) are uncoupled from the phase modulation $\dot{\theta}$.

After the bifurcation equations (22) have been numerically integrated for a given set of parameters and given initial conditions, the state is obtained as $\mathbf{x} \varepsilon \mathbf{x}_{0}+\varepsilon^{2} \mathbf{x}_{2}+O\left(\varepsilon^{3}\right)$. By accounting for Eqs. $\left(17_{1,2}\right)$ and remembering (Eqs. (21)) that $A \quad A_{1}, d_{1} A=$ $\dot{A}=A_{2}$, the state reads

$$
\begin{aligned}
\mathbf{x}= & \frac{1}{2}\left[a \mathbf{u}_{1}+(u+i v) \mathbf{u}_{2}\right] \mathrm{e}^{\mathrm{i}(\omega t+\theta)}+\frac{1}{4} a^{2}\left(\mathbf{z}_{11} \mathrm{e}^{2 \mathrm{i}+(\omega t+\theta)}+\mathbf{z}_{11}\right) \\
& + \text { c.c. }+ \text { higher order terms }
\end{aligned}
$$

The trivial solution $a \quad u \quad v \quad 0$ satisfies the bifurcation equations (22) for any $\boldsymbol{\mu}$, according to the existence of the trivial equilibrium path $\mathbf{x} \quad \mathbf{0} \forall \boldsymbol{\mu}$. Steady state non trivial solutions are obtained by vanishing the right hand member of Eqs. $\left(22_{1,2,3}\right)$. These solutions correspond to periodic motions ( $a$ const) of the original system, of nonlinear frequency $\Omega:=\omega+v / a$. Their stability is gov erned by the variational equations of Eq. (22); in con trast, stability of the trivial solution calls for use of the variation of Eq. (20) since, Eq. (22) 4 becomes singular when $a$ 0. Finally, periodic solutions of Eqs. $\left(22_{1,2,3}\right)$ 
correspond to quasi periodic motions of the original sys tem. Their stability is governed by the Floquet charac teristic exponents.

\section{An automatic procedure for evaluating the bifurcation equation}

In order to show the effectiveness of the proposed method, we illustrate an algorithm which can be used to evaluate numerically the coefficients of the bifurcation equation (20) for a class of systems. The method does not require repetition of the whole procedure for the specific system under study, but only evaluation of the numeri cal coefficients, which is achieved by performing ele mentary operations. In this respect the method is user oriented, in contrast to other methods, such as, for example, the center manifold method, which have not yet been applied to furnish ready to use formulas.

A broad class of $N$ dimensional mechanical systems is considered, having equations:

$\dot{\mathbf{x}}=(\mathbf{A}+\mathbf{B} \boldsymbol{\mu}) \mathbf{x}+\mathbf{c}(\mathbf{x})$

where the matrices $\mathbf{A}$ and $\mathbf{B}$ are constant, $\boldsymbol{\mu}$ is the control parameter vector and the vector $\mathbf{c}(\mathbf{x})$ collects quadratic and cubic nonlinearities. The $i$ th equation (24) therefore reads:

$$
\begin{aligned}
\dot{x}_{i}= & \sum_{i} a_{i j} x_{j}+\sum_{j, k} \mu_{j} b_{i j k} x_{k}+\frac{1}{2} \sum_{j, k} c_{i j k} x_{j} x_{k} \\
& +\frac{1}{6} \sum_{j, k, l} c_{i j k l} x_{j} x_{k} x_{1}+O\left(x^{2} \boldsymbol{\mu}\right)
\end{aligned}
$$

where coefficients $c$ 's are symmetrical with respect to indices $j, k, l$. For this class $\mathbf{F}_{\mathbf{x}}^{0}=\mathbf{A}, \mathbf{F}_{\mathbf{x} \boldsymbol{\mu}}^{0}=\mathbf{B}$. The follow ing functions are defined:

$$
\begin{aligned}
& f(\mathbf{v}, \mathbf{u}, \boldsymbol{\mu}):=\mathbf{v}^{\mathrm{T}} \mathbf{F}_{\mathbf{x} \mu}^{0} \mathbf{u} \mu=\sum_{i, j, k} b_{i j k} v_{i} u_{j} \mu_{k} \\
& g(\mathbf{v}, \mathbf{u}, \mathbf{w}):=\mathbf{v}^{\mathrm{T}} \mathbf{F}_{\mathbf{x x}}^{0} \mathbf{u w}=\sum_{i, j, k} c_{i j k} v_{i} u_{j} w_{k} \\
& h(\mathbf{v}, \mathbf{u}, \mathbf{w}, \mathbf{y}):=\mathbf{v}^{\mathrm{T}} \mathbf{F}_{\mathbf{x x x}}^{0} \mathbf{u w y}=\sum_{i, j, k, l} c_{i j k l} v_{i} u_{j} w_{k} y_{1}
\end{aligned}
$$

The above functions associate a real number with the dummy vectors $\mathbf{v}, \mathbf{u}, \boldsymbol{\mu}, \mathbf{w}$ and $\mathbf{y}$. By appropriately choos ing the arguments, the functions (26) furnish all the quantities necessary to evaluate the coefficients appear ing in the bifurcation equation (20). In particular, by taking $\mathbf{v}$ equal to the canonical vector $\mathbf{e}_{j} \quad\left\{\delta_{i j}\right\}(i, j$ $1,2, \ldots, N)$ they also make it possible to build up the vec tor equations in Appendix B.

The following step by step algorithm is applied.

(a) Proper and generalized right eigenvectors are evalu ated at the bifurcation point $\boldsymbol{\mu} \mathbf{0}$ by solving the equations: $\left(\begin{array}{lllll}\mathbf{A} & \lambda_{0} \mathbf{I}\end{array}\right) \mathbf{u}_{1} \quad \mathbf{0},\left(\begin{array}{lll}\mathbf{A} & \lambda_{0} \mathbf{I}\end{array}\right) \mathbf{u}_{2} \quad \mathbf{u}_{1}$, (A $\left.\quad \lambda_{0} \mathbf{I}\right)^{\mathrm{T}} \mathbf{v}_{2} \quad \mathbf{0},\left(\begin{array}{lll}\mathbf{A} & \lambda_{0} \mathbf{I}\end{array}\right)^{\mathrm{T}} \mathbf{v}_{1} \quad \mathbf{v}_{2}$ and normalizing the solutions according to $\mathbf{v}_{j}^{\mathrm{T}} \mathbf{u}_{k}=\delta_{j k}$. (b) To solve the nonsingular Eq. (B.1 $1_{1}$ ) the known terms of this equation are built up, as

$\mathbf{F}_{\mathbf{x x}}^{0} \mathbf{u}_{1}^{2}=\left\{g\left(\mathbf{e}_{j}, \mathbf{u}_{1}, \mathbf{u}_{1}\right)\right\}$

where the brackets collect the vector coefficients for $j$ running from 1 to $N$. Then $\mathbf{z}_{11}$ is evaluated by solv ing the Equation (B. $1_{1}$ ).

(c) By using arguments similar to step (b), all the non singular equations $\left(B .1_{2-5}\right)$ are solved and $\left(\mathbf{z}_{11}, \mathbf{z}_{11}, \mathbf{z}_{12}, \mathbf{z}_{111}\right)$ are sequentially evaluated.

(d) To solve the singular Eq. (B.16), the known terms of this equation are built up, as

$$
\begin{aligned}
& \mathbf{F}_{\mathbf{x x}}^{0} \mathbf{z}_{11} \mathbf{u}_{1}=\left\{g\left(\mathbf{e}_{j}, \mathbf{z}_{11}, \mathbf{u}_{1}\right)\right\}, \\
& \mathbf{F}_{\mathbf{x x}}^{0} \mathbf{z}_{11} \overline{\mathbf{u}}_{1}=\left\{g\left(\mathbf{e}_{j}, \mathbf{z}_{11}, \overline{\mathbf{u}}_{1}\right)\right\} \\
& \mathbf{F}_{\mathbf{x x}}^{0} \overline{\mathbf{z}}_{11} \overline{\mathbf{u}}_{1}=\left\{g\left(\mathbf{e}_{j}, \overline{\mathbf{z}}_{11}, \mathbf{u}_{1}\right)\right\}, \\
& \mathbf{F}_{\mathbf{x x x}}^{0} \mathbf{u}_{1}^{2} \overline{\mathbf{u}}_{1}=\left\{h\left(\mathbf{e}_{j}, \mathbf{u}_{1}, \mathbf{u}_{1}, \mathbf{u}_{1}\right)\right\} \\
& \left(\mathbf{v}_{2}^{\mathrm{H}} \mathbf{F}_{\mathbf{x x}}^{0} \mathbf{z}_{11} \mathbf{u}_{1}\right) \mathbf{u}_{2}=g\left(\mathbf{v}_{2}, \mathbf{z}_{11}, \mathbf{u}_{1}\right) \mathbf{u}_{2} \\
& \left(\mathbf{v}_{2}^{\mathrm{H}} \mathbf{F}_{\mathbf{x x}}^{0} \mathbf{z}_{11} \overline{\mathbf{u}}_{1}\right) \mathbf{u}_{2}=g\left(\mathbf{v}_{2}, \mathbf{z}_{11}, \overline{\mathbf{u}}_{1}\right) \mathbf{u}_{2} \\
& \left(\mathbf{v}_{2}^{\mathrm{H}} \mathbf{F}_{\mathbf{x x}}^{0} \overline{\mathbf{z}}_{11} \mathbf{u}_{1}\right) \mathbf{u}_{2}=g\left(\mathbf{v}_{2}, \overline{\mathbf{z}}_{11}, \mathbf{u}_{1}\right) \mathbf{u}_{2} \\
& \left(\mathbf{v}_{2}^{\mathrm{H}} \mathbf{F}_{\mathbf{x x x}}^{0} \mathbf{u}_{1}^{2} \overline{\mathbf{u}}_{1}\right) \mathbf{u}_{2}=h\left(\mathbf{v}_{2}, \mathbf{u}_{1}, \mathbf{u}_{1}, \overline{\mathbf{u}}_{1}\right) \mathbf{u}_{2}
\end{aligned}
$$

Then $\mathbf{z}_{111}$ is evaluated by solving equation (B.16) un der the constraint condition $\mathbf{v}_{1}^{\mathrm{T}} \mathbf{z}_{111}=0$.

(e) To solve Eq. $\left(B .1_{7}\right), \mu$ is first set equal to the canon ical vector $\mathbf{e}_{k}$. The known terms then read

$$
\begin{aligned}
\mathbf{F}_{\mathbf{x} \mu}^{0} \mathbf{u}_{1} \mathbf{e}_{k} & =\left\{f\left(\mathbf{e}_{j}, \mathbf{u}_{1}, \mathbf{e}_{k}\right)\right\},\left(\mathbf{v}_{2}^{\mathrm{T}} \mathbf{F}_{\mathbf{x} \mu}^{0} \mathbf{u}_{1} \mathbf{e}_{k}\right) \mathbf{u}_{2} \\
& =f\left(\mathbf{v}_{2}, \mathbf{u}_{1}, \mathbf{e}_{k}\right) \mathbf{u}_{2}
\end{aligned}
$$

By solving the relevant equation, the $k$ th column of the matrix $\mathbf{Z}_{1 \boldsymbol{\mu}}$ is evaluated, provided it is orthogonal to $\mathbf{v}_{1}$, since the operator is singular. By letting $k$ run from 1 to $M$, the whole matrix $\mathbf{Z}_{1 \mu}$ is obtained.

(f) Coefficients $\mathbf{C}$ in Eqs. (20) are finally evaluated by Eqs. (B.2); e.g.

$$
\begin{aligned}
& \mathbf{C}_{2 \boldsymbol{\mu}}=\left\{f\left(\mathbf{v}_{2}, \mathbf{u}_{2}, \mathbf{e}_{k}\right) \quad \mathbf{v}_{2}^{\mathrm{T}} \mathbf{Z}_{1 \boldsymbol{\mu}} \mathbf{e}_{k}\right\} \\
& \text { for } k \quad 1,2, \ldots, M .
\end{aligned}
$$

It should be noted, that the procedure illustrated makes it possible to obtain a bifurcation equation para metric in $\boldsymbol{\mu}$, so that the procedure does not have to be repeated for any choice of $\boldsymbol{\mu}$. Once the algorithm has been implemented, only the coefficients $a_{i j}, b_{i j k}, c_{i j k}$ and $c_{i j k l}$ in Eqs. (25) must be given for any specific system.

\section{Sample system}

\subsection{Model and bifurcation equation}

The structure of Fig. 1a, is analyzed. It consists of two vertical rigid bars of length $l$, constrained at the 


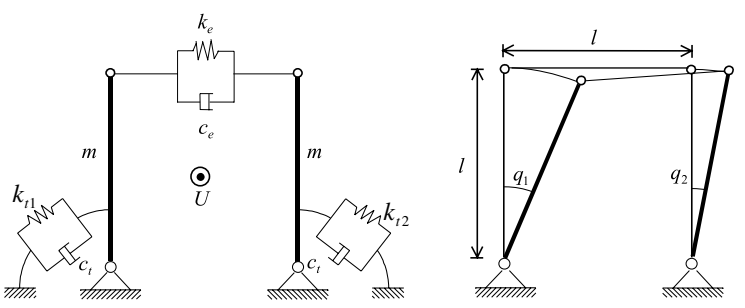

(a)

(b)

Fig. 1. (a) System of two rigid bars under aerodynamic excitation and (b) Lagrangian parameters.

ground by two different linear visco elastic hinges joined at their ends by a linear visco elastic control device, which can have either positive or negative stiffness. The structure is loaded by a fluid flow of uniform veloc ity $U$ orthogonal to the plane of the motion. By assum ing the rotations $q_{i}(i \quad 1,2)$ as Lagrangian parameters (Fig. 1b) and applying the quasi static theory to express the aerodynamic forces [23], the dimensional equations of motion of the system in Fig. 1a, expanded up to the third order, read

$$
\begin{aligned}
& \frac{1}{3} l^{3} m \ddot{q}_{1}+\left[c_{\mathrm{t}}+c_{\mathrm{e}} l^{2}+\frac{1}{6} b\left(c_{\mathrm{d}}+c_{1}^{\prime}\right) l^{3} U \rho\right] \dot{q}_{1} \\
& c_{\mathrm{e}} l^{2} \dot{q}_{2}+\left(k_{t 1}+k_{\mathrm{e}} l^{2}\right) q_{1} \quad k_{\mathrm{e}} l^{2} q_{2} \\
& \frac{1}{2} b \rho\left[\frac{1}{8}\left(2 c_{\mathrm{d}}^{\prime}+c_{1}+c_{1}^{\prime \prime}\right) l^{4} \dot{q}_{1}^{2}\right. \\
& \left.\frac{1}{30 U}\left(3 c_{\mathrm{d}}+3 c_{\mathrm{d}}^{\prime \prime}+c_{1}^{\prime}+c_{1}^{\prime \prime}\right) l^{5} \dot{q}_{1}^{3}\right] \\
& \frac{2}{3} k_{\mathrm{e}} l^{2} q_{1}^{3}+\frac{1}{2} k_{\mathrm{e}} l^{2} q_{1}^{2} q_{2}+\frac{1}{6} k_{\mathrm{e}} l^{2} q_{2}^{3} \\
& c_{\mathrm{e}} l^{2}\left(\begin{array}{lll}
q_{1}^{2} \dot{q}_{1} & \frac{1}{2} q_{1}^{2} \dot{q}_{2} & \frac{1}{2} q_{2}^{2} \dot{q}_{2}
\end{array}\right)=0 \\
& \frac{1}{3} l^{3} m \ddot{q}_{2} \quad c_{\mathrm{e}} l^{2} \dot{q}_{1}+\left[c_{\mathrm{t}}+c_{\mathrm{e}} l^{2}+\frac{1}{6} b\left(c_{\mathrm{d}}+c_{1}^{\prime}\right) l^{3} U \rho\right] \dot{q}_{2} \\
& k_{\mathrm{e}} l^{2} q_{1}+\left(k_{t 2}+k_{\mathrm{e}} l^{2}\right) q_{2} \quad \frac{1}{2} b \rho\left[\frac{1}{8}\left(2 c_{\mathrm{d}}^{\prime}+c_{1}+c_{1}^{\prime \prime}\right)\right. \\
& \left.\times l^{4} \dot{q}_{2}^{2} \quad \frac{1}{30 U}\left(3 c_{\mathrm{d}}+3 c_{\mathrm{d}}^{\prime \prime}+c_{1}^{\prime}+c_{1}^{\prime \prime}\right) l^{5} \dot{q}_{2}^{3}\right] \\
& \frac{2}{3} k_{\mathrm{e}} l^{2} q_{2}^{3}+\frac{1}{2} k_{\mathrm{e}} l^{2} q_{1} q_{2}^{2}+\frac{1}{6} k_{\mathrm{e}} l^{2} q_{1}^{3} \\
& c_{\mathrm{e}} l^{2}\left(\begin{array}{lll}
q_{2}^{2} \dot{q}_{2} & \frac{1}{2} q_{1}^{2} \dot{q}_{1} & \frac{1}{2} \dot{q}_{1} q_{2}^{2}
\end{array}\right)=0
\end{aligned}
$$

In Eqs. (32) $m$ is the mass per unit of length of the bars, $k_{t 1}$ and $k_{t 2}$ are the torsional stiffnesses, $k_{\mathrm{e}}$ is the stiffness of the extensional device, $c_{\mathrm{e}}$ and $c_{\mathrm{t}}$ are the damping coef ficients of the extensional and torsional devices, $\rho$ is the air density, $b$ is a characteristic length of the cross sec tion of the bars, $c_{\mathrm{d}}$ and $c_{1}$ are the non dimensional drag and lift coefficients and the apices denote differentiation with respect to the attack angle. Eqs. (32) are nondimen sionalized by introducing the following quantities:

$$
\begin{aligned}
& \tau=\omega_{1} t ; \quad \omega_{1}^{2}=\frac{3 k_{t 1}}{m l^{3}} ; \quad \xi_{\mathrm{e}}=\frac{3 c_{\mathrm{e}}}{2 m \omega_{1} l} \\
& \xi_{\mathrm{t}}=\frac{3 c_{\mathrm{t}}}{2 m \omega_{1} l^{3}} ; \quad d_{1}=\frac{1}{2}\left(c_{\mathrm{d}}+c_{1}^{\prime}\right) \\
& d_{2}=\frac{3}{16} \frac{\rho b l}{m_{1}}\left(c_{1}^{\prime \prime}+c_{1}+2 c_{\mathrm{d}}^{\prime}\right) ; \\
& d_{3}=\frac{1}{20}\left(\frac{\rho b l}{m_{1}}\right)^{2}\left(c_{1}^{\prime \prime}+c_{1}^{\prime}+3 c_{\mathrm{d}}^{\prime \prime}+3 c_{\mathrm{d}}\right) \\
& \mu=\frac{\rho b}{m \omega_{1}} U ; \quad v=\frac{k_{\mathrm{e}}}{m \omega_{1}^{2} l} ; \quad \eta=\frac{k_{t 1}}{k_{t 2}}
\end{aligned}
$$

The parameters $\mu$ (nondimensional wind velocity), $v$ (nondimensional stiffness of the extensional spring) and $\eta$ (torsional stiffness ratio) are assumed as control parameters, i.e. $\boldsymbol{\mu}:=\{\mu, v, \eta\}$. The parameter $\mu$ will be re ferred to as distinguished parameter, since it represents the intensity of the external load; the remaining two, $v$ and $\eta$, will be referred to as splitting parameters, since they are responsible for the tuning of the critical eigen values. An eigenvalue analysis of the (linearized) equa tions of motion shows that at $\boldsymbol{\mu} \quad \boldsymbol{\mu}_{0}$ a $1: 1$ resonant double Hopf bifurcation occurs, with

$\mu_{0}=\frac{2\left(\xi_{\mathrm{t}}+\xi_{\mathrm{e}}\right)}{d_{1}} ; \quad v_{0}=0 \quad \eta_{0}=1+4 \xi_{\mathrm{e}}+4 \xi_{\mathrm{e}}^{2}$

By defining incremental parameters $\widehat{\boldsymbol{\mu}}:=\boldsymbol{\mu} \quad \boldsymbol{\mu}_{0}$ and omitting the hat, the equations of motion are put in the state form (24), where the dot now denotes differen tiation with respect to the dimensionless temporal scale, $\tau, \mathbf{x}=\left(x_{1}, x_{2}, x_{3}, x_{4}\right)^{\mathrm{T}}=\left(q_{1}, \dot{q}_{1}, q_{2}, \dot{q}_{2}\right)^{\mathrm{T}}$ is the state vector $\mathbf{A}=\left[\begin{array}{c:c:c:c}0 & 1 & 0 & 0 \\ \hdashline-1 & -d_{1} \mu_{0}-2\left(\xi_{t}+\xi_{e}\right) & 0 & 2 \xi_{e} \\ \hdashline 0 & 0 & 0 & 1 \\ \hdashline 0 & 2 \xi_{e} & \eta_{0} & -d_{1} \mu_{0}-2\left(\xi_{t}+\xi_{e}\right)\end{array}\right]$

and

$\mathbf{B} \mu=\left[\begin{array}{c:c:c:c}0 & 0 & 0 & 0 \\ \hdashline-3 v & -d_{1} \mu & 3 v & 0 \\ \hdashline 0 & 0 & 0 & 0 \\ \hdashline 3 v & 0 & \eta-3 v & -d_{1} \mu\end{array}\right]$

are the Jacobian matrix and its first variation evaluated at the bifurcation point $O$, respectively; finally, c $\left(0, c_{1}(\mathbf{x}), 0, c_{2}(\mathbf{x})\right)^{\mathrm{T}}$ is the nonlinearity vector evalu ated at $\boldsymbol{\mu} \mathbf{0}$, where

$$
\begin{array}{lll}
c_{1}=d_{2} x_{2}^{2}+\frac{d_{3}}{\mu_{0}} x_{2}^{3}+2 x_{1}^{2} x_{2} \xi_{\mathrm{e}} & x_{1}^{2} x_{4} \xi_{\mathrm{e}} & x_{3}^{2} x_{4} \xi_{\mathrm{e}} \\
c_{2}=d_{2} x_{4}^{2}+\frac{d_{3}}{\mu_{0}} x_{4}^{3}+2 x_{3}^{2} x_{4} \xi_{\mathrm{e}} & x_{1}^{2} x_{2} \xi_{\mathrm{e}} & x_{2} x_{3}^{2} \xi_{\mathrm{e}}
\end{array}
$$

The following values of the nondimensional parame ters are taken: $\xi_{\mathrm{e}} \quad \xi_{\mathrm{t}} \quad 0.05 ; d_{1} \quad 1.345, d_{2} \quad 0$, 
$d_{3} \quad 1.251$ (corresponding to a square cross section of the bars). Consequently, the critical parameters are $\boldsymbol{\mu}_{0} \quad 0.149, v_{0} \quad 0, \eta_{0} \quad 1.210$ and the associate double eigenvalue is $\lambda_{0} \pm \mathrm{i} 1.049$. The non vanishing coeffi cients in the equations of motion (25) assume the follow ing values: $a_{12} \quad 1, a_{21} \quad 1, a_{24} \quad 0.1, a_{34} \quad 1, a_{42} \quad 0.1$, $a_{43} 1.21 ; b_{221} \quad 0.5, b_{212} 1.35, b_{223} 3.0, b_{421} \quad 3$,

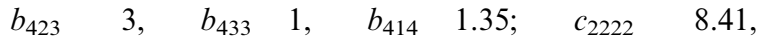

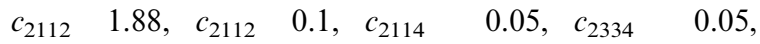
$c_{4444} \quad 1.88, c_{4334} \quad 0.1, c_{4112} \quad 0.05, c_{4233} \quad 0.05$. Proper and generalized eigenvectors are found to be

$\mathbf{u}_{1}=\left\{\begin{array}{c}0.499 \mathrm{i} \\ 0.524 \\ 0.476 \\ 0.499 \mathrm{i}\end{array}\right\}, \quad \mathbf{u}_{2}=\left\{\begin{array}{c}1.0 \\ 0.499 \mathrm{i} \\ 0.454 \mathrm{i} \\ 15.238\end{array}\right\}$,

$\mathbf{v}_{1}=\left\{\begin{array}{c}1.0 \\ 0.062+1.049 \mathrm{i} \\ 1.4231 .154 \mathrm{i} \\ 1.0+1.291 \mathrm{i}\end{array}\right\}, \quad \mathbf{v}_{2}=\left\{\begin{array}{c}0.062 \\ 0.065 \mathrm{i} \\ 0.071 \mathrm{i} \\ 0.062\end{array}\right\}$

Since $d_{2} \quad 0$, quadratic terms in Eqs. (37) are identically zero (i.e. $\mathbf{F}_{\mathbf{x x}}{ }^{0} \quad \mathbf{0}$ ), and therefore the system is symmet ric. This entails the vanishing of some terms in Eqs. (B.1). By solving these equations the following solutions are found:

$$
\begin{aligned}
& \mathbf{z}_{11}=\mathbf{0}, \quad \mathbf{z}_{12}=\mathbf{0}, \quad \mathbf{z}_{11}=\mathbf{0}, \quad \mathbf{z}_{12}=\mathbf{0} \\
& \mathbf{z}_{111}=\left\{\begin{array}{c}
0.133 \\
0.418 \mathrm{i} \\
0.127 \mathrm{i} \\
0.399
\end{array}\right\} \\
& \mathbf{z}_{111}=\left\{\begin{array}{c}
32.757+25.849 \mathrm{i} \\
27.112+34.370 \mathrm{i} \\
24.722+0.825 \mathrm{i} \\
0.859 \quad 25.929 \mathrm{i}
\end{array}\right\} \\
& \mathbf{Z}_{1 \mu}=\left[\begin{array}{cccc}
7.197 & 5.709 \mathrm{i} & 1.423 & 26.133 \mathrm{i} \\
5.988 & 7.553 \mathrm{i} & 27.418 & 1.677 \mathrm{i} \\
5.443 & 0.168 \mathrm{i} & 10.681+11.218 \mathrm{i} \\
0.175+5.709 \mathrm{i} & 11.849+11.199 \mathrm{i}
\end{array}\right. \\
& \left.\begin{array}{c}
0.1250 .171 \mathrm{i} \\
0.150+0.131 \mathrm{i} \\
0.003+0.119 \mathrm{i} \\
0.125+0.010 \mathrm{i}
\end{array}\right]
\end{aligned}
$$

Finally, the coefficients defined by Eqs. (B.2) are evalu ated, and bifurcation equation (20) built up

$$
\begin{aligned}
& \ddot{A}=\left[\begin{array}{ll}
\mathrm{i} 0.00414 \mu+(0.0088 \mathrm{i} 0.1845) v
\end{array}\right. \\
& 0.0293 \eta] A+[2.1271 \mu+(1.8194 \\
& +\mathrm{i} 2.2094) v+\mathrm{i} 0.0478 \eta] \dot{A}+(0.0023 \\
& +\mathrm{i} 0.2335) A^{2} A+(19.5833+\mathrm{i} 0.0669) A A \dot{A} \\
& +(1.5764+\mathrm{i} 0.0110) A^{2} \dot{A}
\end{aligned}
$$

\subsection{Numerical results}

The mechanical behavior of the system around the bifurcation point is investigated. The three dimensional linear stability diagram of the trivial solution is plotted in Fig. 2a (exact surface) and 2b (approximate surface), obtained from an eigenvalue analysis of the Jacobian matrix of Eq. (32) or (40), respectively. The surfaces are the loci of simple Hopf bifurcations; at the crossing of two branches non resonant double Hopf bifurcations occur, while at the singular points $O$ and $W$, the two bifurcations are in 1:1 resonant condition. The approxi mate locus is tangent to the exact locus at point $O$, as shown in Fig. 2c. However, it cannot describe either the loop or the coexisting bifurcation point $W$. The investigation must therefore be confined to a small re gion around $O$.

By sectioning the $3 \mathrm{D}$ plots of Fig. $2 \mathrm{a}$ and $\mathrm{b}$ with planes $v$ const and $\eta$ const (i.e. by fixing one of the two splitting parameters and letting the second vary to gether with the distinguished parameter $\mu$ ), the plots in Fig. 3 are obtained, where exact and approximate boundaries are compared. Two Hopf curves $\mathscr{H}_{1}$ and $\mathscr{H}_{2}$ are illustrated, from which periodic solutions in the state variables $\mathbf{x}$ (i.e. steady state amplitude solu tions $a \quad$ const) bifurcate. When $v$ const (Fig. 3a c), the accordance between the two sets is good for $\eta>0$; in contrast, due to the existence of a vertical asymptote, no approximate curves exist when $\eta<0$. In contrast, when $\eta$ const $>0$ (Fig. $3 \mathrm{~d} \mathrm{f}$ ), the two sets have the same qualitative aspect. In particular, at $\eta \quad 0$ (Fig. $3 \mathrm{~d}$ ), curves $\mathscr{H}_{1}$ and $\mathscr{H}_{2}$ are almost tangent at the origin. Due to the fast variation of the curvature of the exact boundaries, there exists a region (shaded in the figure) in which the number of the bifurcation points encoun tered along a path $v$ const is different in the two cases (namely, four for the exact, two for the approximate). However, when $\eta$ exceeds the value $\eta \eta^{*} 0.0026$ (Fig. 3e) where $\mathscr{H}_{2}$ has a vertical tangent at $O$, this dis crepancy disappears (see Fig. 3f).

The amplitude $a$ of the periodic solutions bifurcating from the $\mathscr{H}_{1}$ and $\mathscr{H}_{2}$ boundaries of Fig. 3a,b,d,e are found as equilibrium points of the bifurcation equation (40); they are illustrated in the following (qualitative) Figs. 4 7, respectively (bifurcation diagrams). Fig. 4 refers to $v \quad 0$. The approximate boundaries in Fig. 4a limit four regions $\mathscr{R}_{i}$ in which $i \quad 0,1,2,3$ periodic solu tions exist (in addition to the trivial one). As shown in Fig. $4 \mathrm{~b}$, two branches bifurcate from the positive $\eta$ half axis, each dying on a distinct $\mu$ half axis. Due to the fold exhibited, by the two surfaces close to the $\mu$ axis (see the sections $\mu$ const in Fig. 4c), more periodic solutions than surfaces coexist in the shaded region of Fig. 4a. This also appears from the cross sections $\eta$ const of Fig. 4d, where the dashed curves refer to $\eta \quad 0$ and are produced by the folds. 


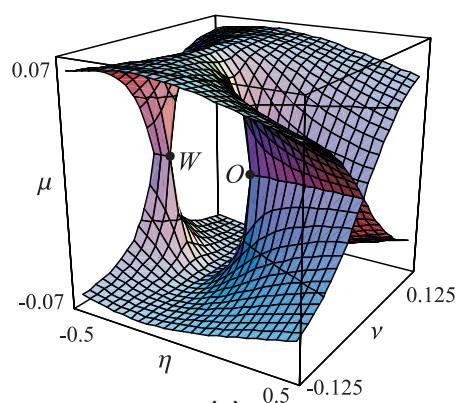

(a)

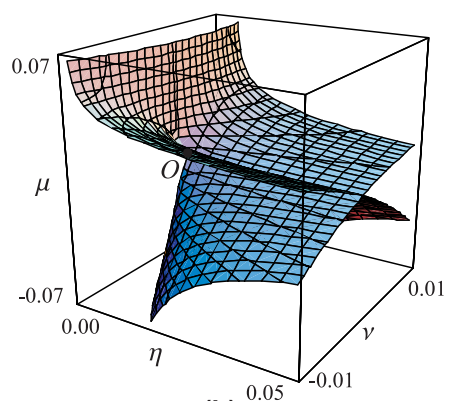

(b)

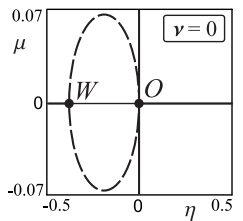

(c)

Fig. 2. Linear stability diagram of the trivial solution: (a) exact solution; (b) approximate solution and (c) section at $v=0$; continuous line: approximate solution and dashed line: exact solution.

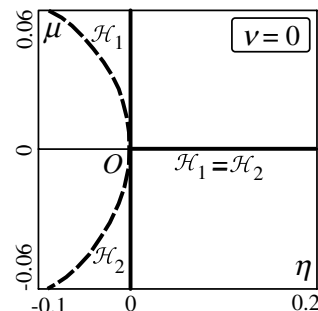

(a)

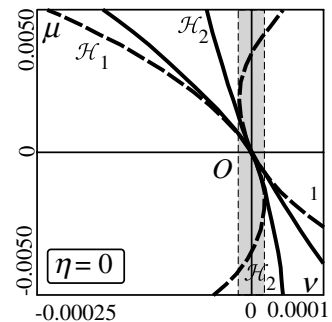

(d)

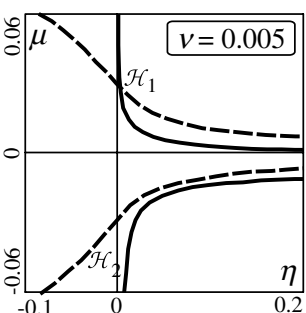

(b)

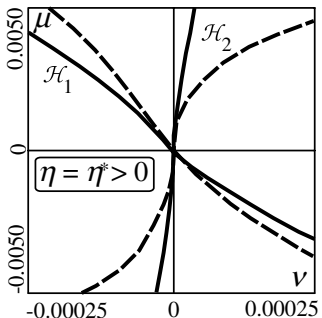

(e)

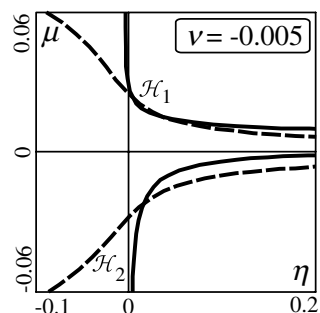

(c)

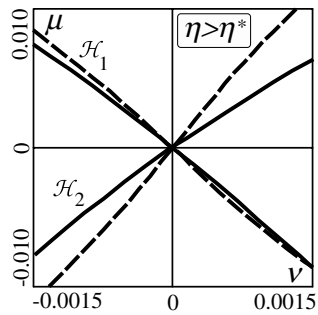

(f)

Fig. 3. Sections of the linear stability diagram: (a) (c) $v=$ const; (d) (f) $\eta=$ const; continuous line: approximate solutions, dashed line: exact solutions.

When $v \neq 0$ (Fig. 5) the coalescence between $\mathscr{H}_{1}$ and $\mathscr{H}_{2}$ is destroyed. Again, the folds of the surfaces deter mine coexisting solutions in the shaded regions of Fig. $5 \mathrm{a}$. The whole scenario appears as a perturbation of that of Fig. 4, produced by the splitting parameter $v$.

Let us now examine the bifurcation diagram for $\eta \quad 0$ (Fig. 6). The boundaries $\mathscr{H}_{1}$ and $\mathscr{H}_{2}$ are shown in Fig. 6a. From them, the periodic solutions sketched in Fig. 6b, bifurcate. They coexist in the shaded region of Fig. 6a, as also appears from the sections of Fig. 6c. In addition to the two solutions, a third periodic solution is found, represented by the upper surface in Fig. 6b. This latter surface does not bifurcate from any curve of the $a \quad 0$ plane. However, as will become evident below, this is an erroneous result due to bad approximation of the exact boundaries, as already discussed for Fig. 3d. Accordingly, this upper periodic solution exists only in the range $0<\eta<\eta^{*}$.

When $\eta>\eta^{*}$ the bifurcation diagram changes as illustrated in Fig. 7. The main difference in comparison with Fig. 6 is represented by the disappearance of the upper periodic solution. Other aspects remain qualita tively unmodified.

To describe completely the scenario around the bifur cation point, quasi periodic (more precisely bi periodic) solutions are sought. These bifurcate from the periodic solutions through Neimark bifurcations (also called sec ondary Hopf bifurcations). In the amplitude representa tion of the motion furnished by Eqs. (22), quasi periodic motions ( $a$ periodic) originate from periodic motions (a const) through Hopf bifurcations. A Jacobian eigen value analysis is therefore performed along the branches 


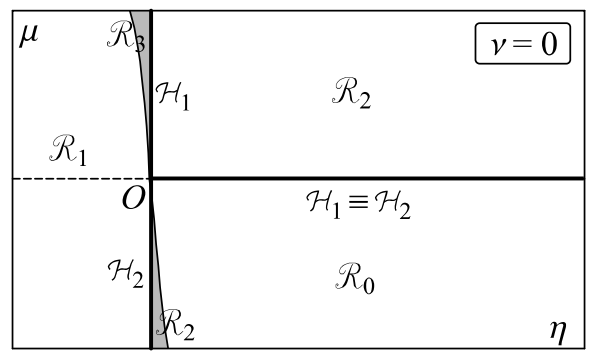

(a)

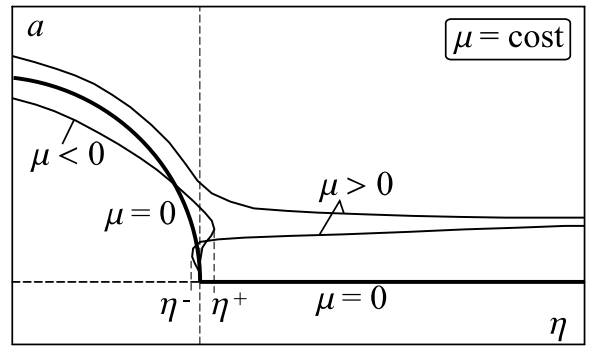

(c)

Fig. 4. Bifurcation diagram for $v=0$ : (a), (c), (d) cross sections and (b) 3D view.

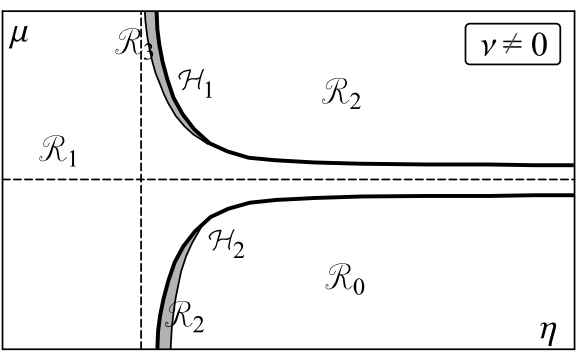

(a)

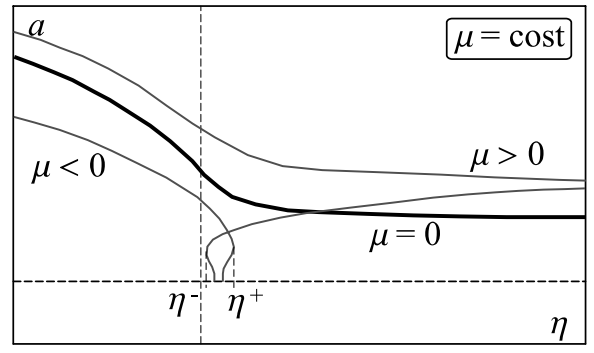

(c)

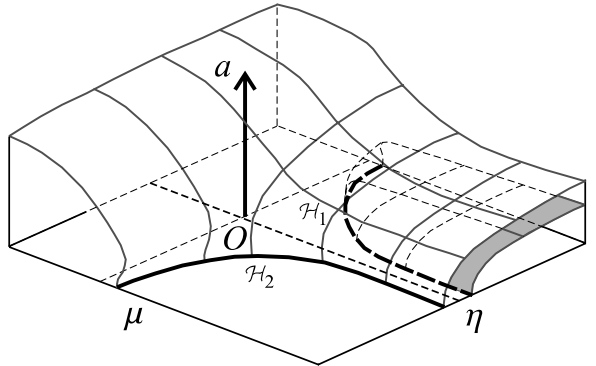

(b)

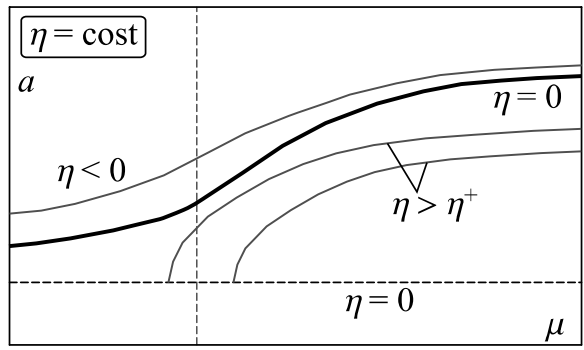

(d)

Fig. 5. Bifurcation diagram for $v=$ const $\neq 0$ : (a), (c), (d) cross sections and (b) $3 \mathrm{D}$ view.

$a$ const by varying one control parameter, and Hopf bifurcations are detected. By following a numerical pro cedure (implemented in the program AUTO ${ }^{\circledR}$ ), the curves of min max modulating amplitude are then built up. The results of the analysis are illustrated in
Fig. 8. In the upper part of the figure (Fig. 8a) the approximate $\mathscr{H}_{1}$ and $\mathscr{H}_{2}$ boundaries of Fig. 3 are dis played (heavy lines). In addition, Neimark boundaries $\mathscr{N}$ are indicated (thin lines), together with homoclinic boundaries $\mathscr{H}_{O M}$ (dashed lines), where the limit cycles 


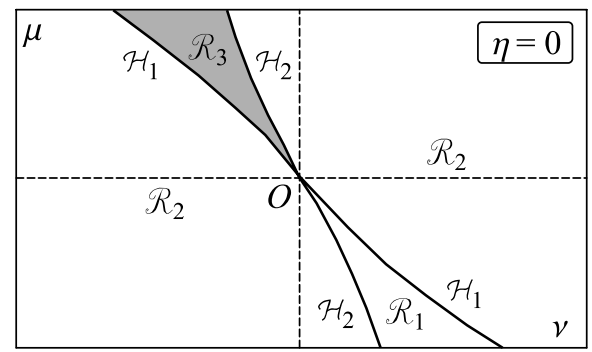

(a)

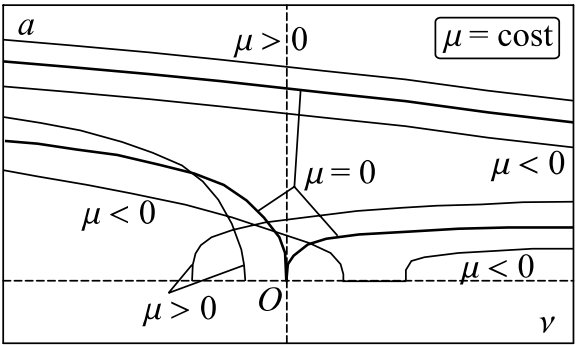

(c)

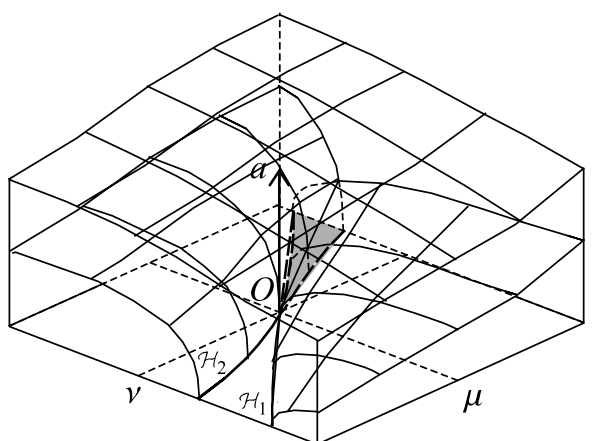

(b)

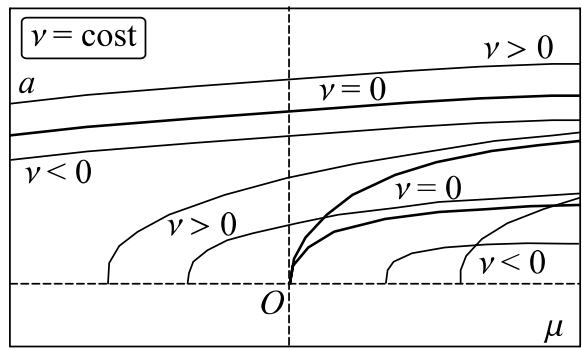

(d)

Fig. 6. Bifurcation diagram for $\eta=0$ : (a), (c), (d) cross sections and (b) 3D view.

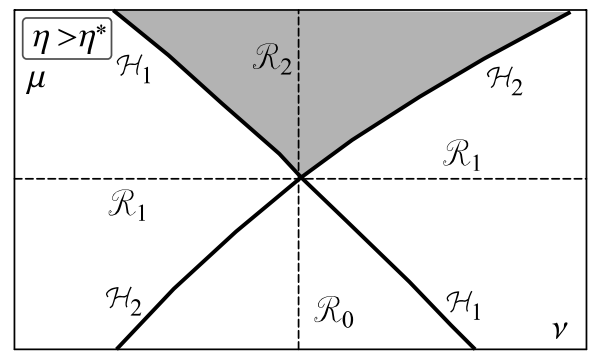

(a)

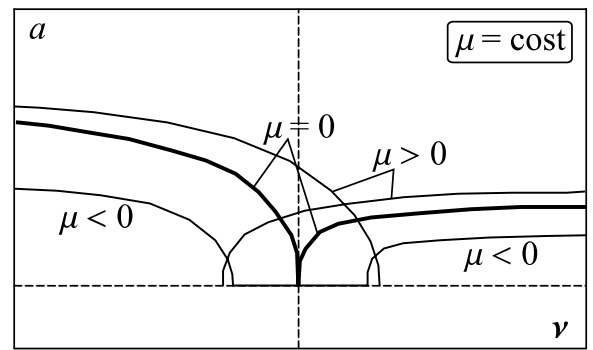

(c)

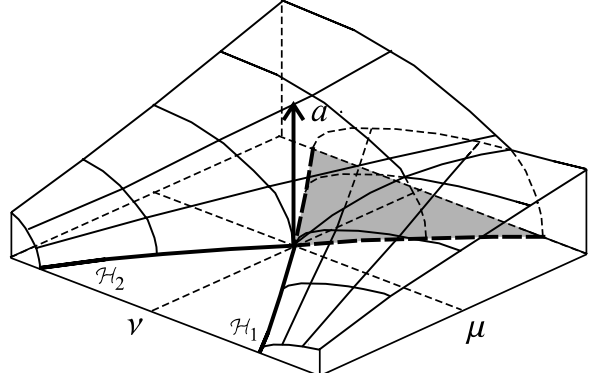

(b)

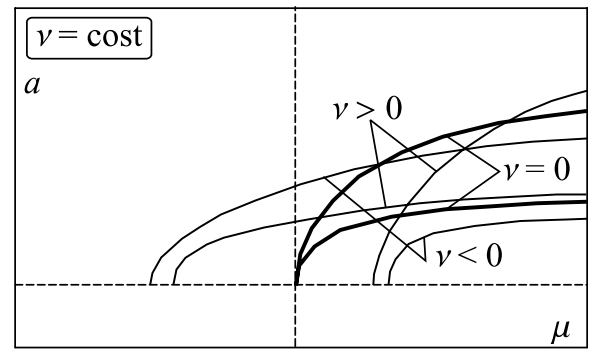

(d)

Fig. 7. Bifurcation diagram for $\eta=$ const $>\eta^{*}$ : (a), (c), (d) cross sections and (b) $3 \mathrm{D}$ view.

for the amplitudes (quasi periodic solutions) disappear after colliding with a saddle (periodic solution). In each region the type of attractor is indicated, $P$ for periodic and $Q$ for quasi periodic motions. In Fig. 8a six paths (I VI) are marked, along which the bifurcation diagrams of the lower part of the figure (Fig. 8b) are 

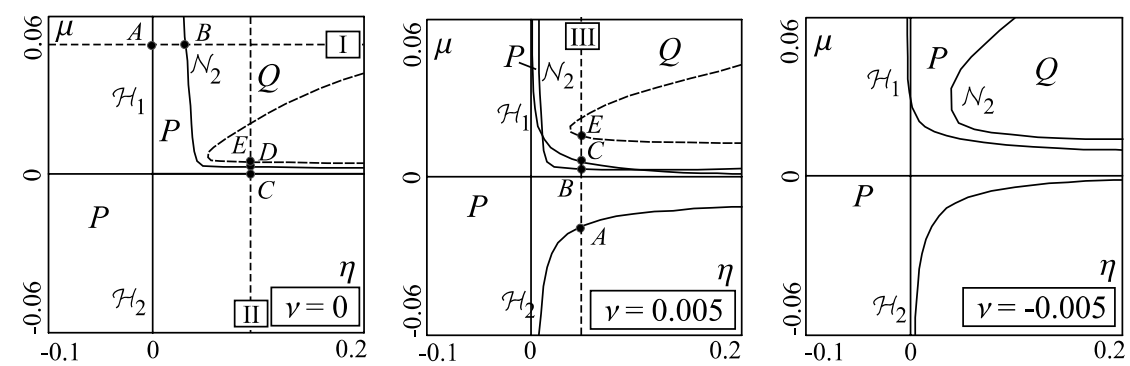

(a)
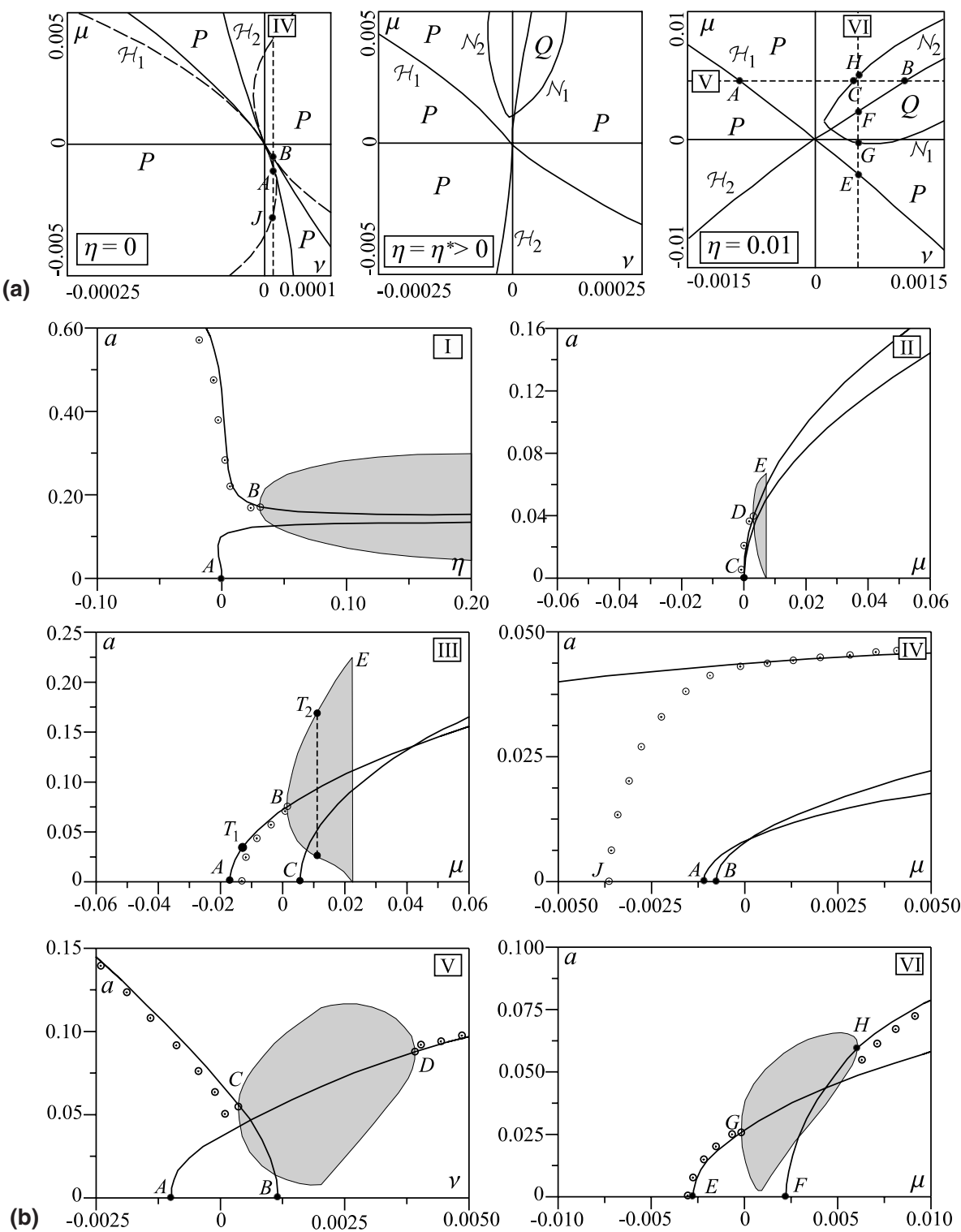

Fig. 8. Bifurcation diagrams along different paths: (a) periodic $\left(\mathscr{H}_{i}\right)$ solutions, quasi periodic $\left(\mathscr{N}_{i}\right)$ solutions, homoclinic boundaries $\left(\mathscr{H}_{O M}\right)$, periodic attractor $(P)$, quasi periodic attractor $(Q)$; (b) amplitudes of periodic solutions (stable heavy lines, unstable thin lines), quasi periodic solutions (shaded regions), exact solutions (circles).

obtained. Here, heavy lines denote stable periodic solu tions, thin lines unstable periodic solutions, and shaded regions the values assumed by the amplitude in quasi periodic motions. Finally, small circles denotes exact 


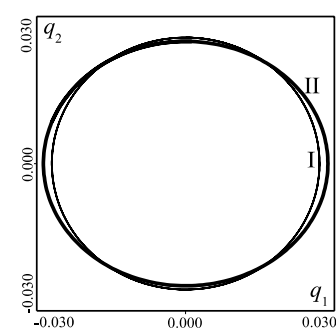

(a)

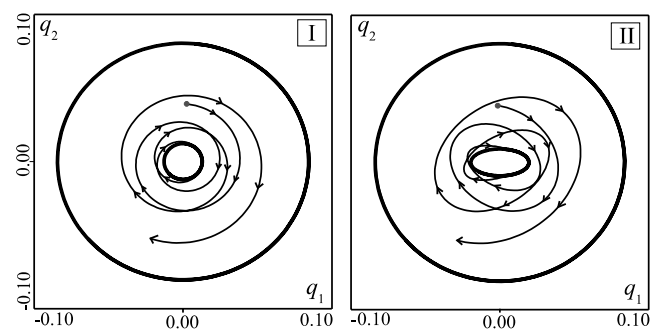

(b)

Fig. 9. Projection of trajectories onto the configuration plane: (I) first order; (II) second order; (a) periodic motion $T_{1}$ in Fig. $8 \mathrm{~b}$ and (b) quasi periodic motion $T_{2}$ in Fig. 8 b.

numerical results drawn by integration of the equations of motion (32). Along path I, an unstable periodic mo tion bifurcates at point $A$ (lying on a Hopf boundary) from the trivial solution. A second branch of stable peri odic solutions loses stability at point $B$ (on the Neimark curve) and gives rise to stable quasi periodic motions. Along path II, at $C$, where the two Hopf boundaries coalesce, two distinct paths arise, one stable and the other unstable. The stable path bifurcates in a quasi periodic solution at $D$ (on the $\mathscr{N}$ curve). However, it disappears at $E$ (on the $\mathscr{H}_{O M}$ curve), after colliding with the trivial solution. Along path III, a stable and an unstable periodic motion bifurcate from the trivial solution at points $A$ and $C$, respectively. The first one undergoes a Neimark bifurcation at $B$, after which the quasi periodic solution disappears at $E$ as a consequence of colliding with the trivial solution. Along path IV two unstable periodic solutions are found originating from $a \quad 0$ at points $A$ and $B$. However, a third upper solution is found, not bifurcating from any point. The numerical results, obtained by integration of Eqs. (32), confirmed the existence of this solution, bifurcating from $a \quad 0$ at point $J$. The approximation is excellent for sufficiently large $\mu$ 's, but the perturbation solution is unable to de scribe this bifurcation. The discrepancy is explained by the fact, already commented in Fig. 3d, that two bifurca tion points are lost, due to the bad approximation of the $\mathscr{H}$ boundaries. Along path $V$, the bifurcated solutions at $A$ and $B$ are initially unstable. They become stable at points $D$ (not shown in Fig. 8a) and $C$, respectively, where stable quasi periodic motions arise. The latter match each other and form a closed loop. A similar phe nomenon is observed along path VI. However, one path is originally stable, while the other is unstable. After Neimark bifurcations they change stability, while quasi periodic solutions merge themselves.

Finally, Fig. 9 represents the projection of some tra jectories onto the configuration $\left(q_{1}, q_{2}\right)$ plane. They were obtained by using Eqs. (23). Fig. 9a refers to the periodic motion $T_{1}$ marked along path III in Fig. 8b. The thin line represents the first order approximation, depending only on the proper vector $\mathbf{u}_{1}$, while the heavy line repre sents the second order approximation, accounting also for the generalized eigenvector $\mathbf{u}_{2}$. This, in the case examined, slightly modifies the length of the ellipsis, but does not entail any rotations, differently from find ings of a similar problem dealt within [16]. It should be noted that double harmonics are absent in the sec ond order solution, due to the symmetry of the system. In Fig. 9b a quasi periodic motion is illustrated, refer ring to point $T_{2}$ marked in Fig. 8b. Both the first and second order approximations are displayed in the figure. The trajectories are spirals confined between the $a_{\text {min }}$ and $a_{\max }$ amplitudes. Second order terms significantily affect only the former.

\section{Conclusions}

An algorithm based on the Multiple Scale Method has been developed to analyze 1:1 resonant $m$ Hopf bifurcations in autonomous discrete systems. The bifur cation occurs when, for certain critical values of the con trol parameters, the Jacobian matrix at an equilibrium point admits $m$ coincident eigenvalues with zero real part. Such a bifurcation is structurally stable in a family of systems depending on $M:=2 m \quad 1$ parameters, where $M$ is the linear codimension of the bifurcation. Since the Jacobian matrix is defective, only one proper eigenvector exists at the critical point, so that the eigen space must be completed by generalized eigenvectors. By exploiting a formal analogy with sensitivity analysis of nilpotent (defective) matrices, a perturbation method has been developed, that is able to furnish a bifurcation equation governing the essential dynamics around the bifurcation. It turns out to be an $m$ th order differential equation in the complex amplitude of the unique critical eigenvector. The method calls for the use of fractional powers expansions of both state variables and time, as opposed to integer power expansions used for non defective bifurcations. In order to improve the efficiency of the method by avoiding trivial steps, even series are used for time and even o complete series for the state var iables, depending on whether the multiplicity $m$ of the 
critical eigenvalue is even or odd. This procedure is sug gested by the analogous sensitivity problem when the perturbation is of singular type (i.e. it belongs to the range of the operator). This circumstance always occurs in the Hopf bifurcation problem, since quadratic non linearities do not produce resonant terms.

The method has then been specialized for a 1:1 reso nant double Hopf bifurcation ( $m \quad 2$ ) and a step by step algorithm has been described to evaluate the coefficients of the bifurcation equation by starting directly from the coefficients of the nonlinear equations of motion, thus avoiding the need to follow the whole asymptotic proce dure for each specific problem. The relevant second order bifurcation equation in a complex quantity is equivalent to three real first order equations, uncoupled from a fourth one, so that the original system is reduced to a three dimensional system. By numerically solving this equation, the qualitative behaviour of the system can be studied in a three dimensional parameter space around the bifurcation point. By referring to a sample mechanical system, where the dynamical instability is triggered by aerodynamic forces, the complete scenario has been built up. The dynamics around a defective dou ble Hopf bifurcation have been found very rich, consist ing in periodic and quasi periodic solutions, suffering homoclinic bifurcations occurring on certain codimen sion 1 manifolds. The results furnished by the perturba tion method have been found agree well with numerical solutions obtained from direct numerical integrations of the equations of motion.

\section{Acknowledgment}

This research was supported by the Italian Ministry for Universities and Scientific Research MIUR (Cofin 200304 funds).

\section{Appendix A. The sensitivity analysis analogy}

The expanded equations of motion (4) can also be written as:

$\left[\mathbf{F}_{\mathbf{x}}^{0}+\varepsilon\left(1 / 2 \mathbf{F}_{\mathbf{x} \mathbf{x}}^{0} \hat{\mathbf{x}}+\mathbf{F}_{\mathbf{x} \boldsymbol{\mu}}^{0} \boldsymbol{\mu}\right)+\varepsilon^{2}\left(1 / 6 \mathbf{F}_{\mathbf{x x x}}^{0} \hat{\mathbf{x}}^{2}+\cdots\right) \quad D\right] \hat{\mathbf{x}}=\mathbf{0}$

where the change of variable $\mathbf{x} \rightarrow \varepsilon \hat{\mathbf{x}}$ is introduced and $D \mathrm{~d} / \mathrm{d} t$ is posed. In this form, the equations resemble a perturbed linear eigenvalue problem, namely

$\left[\left(\mathbf{A}_{0}+\varepsilon \mathbf{A}_{1}+\varepsilon^{2} \mathbf{A}_{2}+\cdots\right) \quad \lambda(\varepsilon)\right] \mathbf{w}(\varepsilon)=\mathbf{0}$

in which the eigenvalue $\lambda$ takes the place of the operator $D$. Eq. (A.2) governs the so called sensitivities of the eigenpairs $(\lambda, \mathbf{w})$ and appears, for example, in the analy sis of stability of an equilibrium path, along which $\lambda_{0}:=\lambda(0)$ is critical. In fact, a nonlinear problem could never be analogous to a linear one; in contrast, this anal ogy holds in a perturbation perspective, in which the nonlinear problem is transformed to become a sequence of linear problems. Therefore, once $\hat{\mathbf{x}}$ has been expanded in a series of $\varepsilon$, the matrices in Eq. (A.1) must be consid ered not as unknowns, but rather as known terms fur nished by the lower order approximations. Indeed, these matrices are $\varepsilon$ dependent, so that a strict analogy holds if and only if the matrices $\mathbf{A}_{k}$ in Eq. (A.2) also depend on $\varepsilon$. However, this aspect can be ignored, since it is unessential to the analysis to be developed here.

Let us assume that both the matrices $\mathbf{F}_{\mathbf{x}}{ }^{0}$ and $\mathbf{A}_{0}$ ad mit a defective eigenvalue $\lambda_{0} \quad \pm \mathrm{i} \omega$ of algebraic multi plicity $m$ and geometrical multiplicity 1 . It is known [19] that problem (A.2) is solved by a complete $\varepsilon^{1 / m}$ series expansion of both the eigenvalue $\lambda$ and the eigenvector w. By exploiting the analogy, the same expansions are used for the operator $D$ and the state variables $\hat{\mathbf{x}}$. To make the discussion easier, specific values $m \quad 3,4$ are considered.

\section{Even $m \quad 4$ case}

The two problems are shown in Table 1. The sensitiv ity analysis is discussed first. Using the series expansions of Eqs. (a), the perturbation equations (b) are drawn. Eq. $\left(b_{1}\right)$ admits the solution $\left(c_{1}\right)$; Eq. $\left(b_{2}\right)$ can be solved for any $\lambda_{1}$, since its known term $\mathbf{u}_{1}$ belongs to the range of the operator, and furnishes the solution $\left(\mathrm{c}_{2}\right)$, with $\lambda_{1}$ still indeterminate. Similarly, Eq. $\left(b_{3,4}\right)$ admits the solu tion $\left(c_{3,4}\right)$, with arbitrary $\lambda_{1}, \lambda_{2}$ and $\lambda_{3}$. By proceeding to higher orders, a solvability condition is first required at the $\varepsilon$ order, where the highest element of the chain $u_{4}$ ap pears together with the perturbation $\mathbf{A}_{1} \mathbf{w}_{0}$. By requiring orthogonality to $\mathbf{v}_{4}$, the degree four algebraic equation $\left(\mathrm{d}_{1}\right)$ in the unknown $\lambda_{1}$ is drawn, from which $m \quad 4$ roots are found (the so called first order sensitivities of the multiple eigenvalue $\lambda_{0}$ ). The relevant solution $\mathbf{w}_{4}$ (Eq. $\left(c_{4}\right)$ ) depends on the sensitivities $\lambda_{2}, \lambda_{3}$ and $\lambda_{4}$, still un known; it also contains a particular solution $\hat{\mathbf{w}}_{4}$ to the problem $\left(\begin{array}{lll}\mathbf{A}_{0} & \lambda_{0} \mathbf{I}\end{array}\right) \hat{\mathbf{w}}_{4}=\lambda_{1}^{m} \quad \mathbf{A}_{1} \mathbf{u}_{1}$, which can be ren dered unique by enforcing a suitable normalization con dition, e.g. $\mathbf{v}_{1}^{\mathrm{H}} \hat{\mathbf{w}}_{4}=0$. At $\varepsilon^{5 / 4}$ order, in contrast, a linear equation $\left(d_{2}\right)$ in $\lambda_{2}$ is found, from which one value of $\lambda_{2}$ is drawn for each of the $m \quad 4$ first order sensitivities. Similarly, at higher orders, linear equations in $\lambda_{3}, \lambda_{4}$, ... follows. The coefficients of series (a) are thus evalu ated. It can be seen that the left members of the solvabil ity equations (d) are the monomials of the expansion of the $m$ th power of $\Delta \lambda:=\lambda \quad \lambda_{0}$. It therefore seems con venient to combine all the equations (d) in a unique alge braic equation (e) of degree $m \quad 4$. This can be referred to as the reconstituted sensitivity equation.

As a special case, if the perturbation $\mathbf{v}_{4}^{\mathrm{H}} \mathbf{A}_{1} \mathbf{u}_{1}$ is equal to zero (singular perturbation), first order solvability equation $\left(\mathrm{d}_{1}\right)$ furnishes $\lambda_{1} \quad 0$. As a consequence, higher order solvability conditions $\left(\mathrm{d}_{2,3,4}\right)$ identically vanish. 
Table 1

Eigenpair sensitivity and bifurcation analysis: $m=4$

$\mathbf{w} \quad \mathbf{w}_{0}+\varepsilon^{1 / 4} \mathbf{w}_{1}+\varepsilon^{1 / 2} \mathbf{w}_{2}+$

$\lambda \quad \lambda_{0}+\varepsilon^{1 / 4} \lambda_{1}+\varepsilon^{1 / 2} \lambda_{2}+$

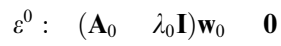

$$
\begin{aligned}
& \varepsilon^{1 / 4}: \quad\left(\begin{array}{lll}
\mathbf{A}_{0} & \left.\lambda_{0} \mathbf{I}\right) \mathbf{w}_{1} & \lambda_{1} \mathbf{w}_{0}
\end{array}\right. \\
& \varepsilon^{1 / 2}:\left(\begin{array}{lll}
\mathbf{A}_{0} & \lambda_{0} \mathbf{I}
\end{array}\right) \mathbf{w}_{2} \quad \lambda_{1} \mathbf{w}_{1}+\lambda_{2} \mathbf{w}_{0} \\
& \varepsilon^{3 / 4}:\left(\begin{array}{lll}
\mathbf{A}_{0} & \lambda_{0} \mathbf{I}
\end{array}\right) \mathbf{w}_{3} \quad \lambda_{1} \mathbf{w}_{2}+\lambda_{2} \mathbf{w}_{1} \\
& +\lambda_{3} \mathbf{w} 0
\end{aligned}
$$$$
\varepsilon: \quad\left(\begin{array}{lll}
\mathbf{A}_{0} & \lambda_{0} \mathbf{I}
\end{array}\right) \mathbf{w}_{4} \quad \lambda_{1} \mathbf{w}_{3}+\lambda_{2} \mathbf{w}_{2}
$$$$
+\lambda_{3} \mathbf{w}_{1}+\lambda_{4} \mathbf{w}_{0} \quad \mathbf{A}_{1} \mathbf{w}_{0}
$$

$$
\begin{aligned}
& \varepsilon^{5 / 4}: \quad\left(\begin{array}{lll}
\mathbf{A}_{0} & \lambda_{0} \mathbf{I}
\end{array}\right) \mathbf{w}_{5} \quad \lambda_{1} \mathbf{w}_{4}+\lambda_{2} \mathbf{w}_{3} \\
& +\lambda_{3} \mathbf{w}_{2}+\quad+\lambda_{5} \mathbf{w}_{0} \quad \mathbf{A}_{1} \mathbf{w}_{1}
\end{aligned}
$$

$\varepsilon^{3 / 2}: \quad\left(\begin{array}{lll}\mathbf{A}_{0} & \left.\lambda_{0} \mathbf{I}\right) \mathbf{w}_{6} & \lambda_{1} \mathbf{w}_{5}+\lambda_{2} \mathbf{w}_{4}\end{array}\right.$

$$
+\quad+\lambda_{6} \mathbf{w}_{0} \quad \mathbf{A}_{1} \mathbf{w}_{2}
$$

$$
\varepsilon^{7 / 4}: \quad\left(\begin{array}{lll}
\mathbf{A}_{0} & \left.\lambda_{0} \mathbf{I}\right) \mathbf{w}_{7} & \lambda_{1} \mathbf{w}_{6}+\lambda_{2} \mathbf{w}_{5}
\end{array}\right.
$$$$
+\quad+\lambda_{7} \mathbf{w}_{0} \quad \mathbf{A}_{1} \mathbf{w}_{3}
$$

$\begin{array}{lll}\varepsilon^{2}: & \left(\begin{array}{lll}\mathbf{A}_{0} & \lambda_{0} \mathbf{I}\end{array}\right) \mathbf{w}_{8} & \lambda_{1} \mathbf{w}_{7}+\quad \lambda_{0} \mathbf{w}_{8}\end{array}$

$\mathbf{A}_{1} \mathbf{w}_{4}$

$\mathbf{A}_{2} \mathbf{w}_{0}$

$$
\begin{array}{rll}
\varepsilon^{0}: & \mathbf{w}_{0} & \mathbf{u}_{1} \\
\varepsilon^{1 / 4}: & \mathbf{w}_{1} & \lambda_{1} \mathbf{u}_{2} \\
\varepsilon^{1 / 2}: & \mathbf{w}_{2} & \lambda_{1}^{2} \mathbf{u}_{3}+\lambda_{2} \mathbf{u}_{2} \\
\varepsilon^{3 / 4}: & \mathbf{w}_{3} & \lambda_{1}^{3} \mathbf{u}_{4}+2 \lambda_{1} \lambda_{2} \mathbf{u}_{3} \\
& +\lambda_{3} \mathbf{u}_{2}
\end{array}
$$$$
\varepsilon: \quad \mathbf{w}_{4} \quad \hat{\mathbf{w}}_{4}+3 \lambda_{1}^{2} \lambda_{2} \mathbf{u}_{4}
$$$$
+\left(\lambda_{2}^{2}+2 \lambda_{1} \lambda_{3}\right) \mathbf{u}_{3}+\lambda_{4} \mathbf{u}_{2}
$$

$$
\begin{aligned}
\varepsilon^{5 / 4}: \quad \mathbf{w}_{5} & \hat{\mathbf{w}}_{5}+3\left(\lambda_{1}^{2} \lambda_{3}+\lambda_{1} \lambda_{2}^{2}\right) \mathbf{u}_{4} \\
+ & 2\left(\lambda_{2} \lambda_{3}+\lambda_{1} \lambda_{4}\right) \mathbf{u}_{3} \\
+ & \lambda_{5} \mathbf{u}_{2}
\end{aligned}
$$

$$
\begin{aligned}
\varepsilon^{3 / 2}: \quad \mathbf{w}_{6} & \hat{\mathbf{w}}_{6}+\left(\lambda_{2}^{3}+3 \lambda_{1}^{2} \lambda_{4}\right) \mathbf{u}_{4} \\
+ & \left(\lambda_{3}^{2}+2 \lambda_{2} \lambda_{4}+2 \lambda_{1} \lambda_{5}\right) \mathbf{u}_{3} \\
+ & \lambda_{6} \mathbf{u}_{2}
\end{aligned}
$$

$$
\begin{array}{lc}
\mathbf{x} & \varepsilon\left(\mathbf{x}_{0}+\varepsilon^{1 / 4} \mathbf{x}_{1}+\varepsilon^{1 / 2} \mathbf{x}_{2}+\quad\right) \\
\mathrm{d} / \mathrm{d} t & d_{0}+\varepsilon^{1 / 4} d_{1}+\varepsilon^{1 / 2} d_{2}+
\end{array}
$$

$$
\begin{aligned}
& \varepsilon: \quad \mathbf{x}_{0} \quad A \mathbf{u}_{1} \mathrm{e}^{\mathrm{i} \omega t_{0}} \\
& \varepsilon^{1+1 / 4}: \quad \mathbf{x}_{1} \quad d_{1} A \mathbf{u}_{2} \mathrm{e}^{\mathrm{i} \omega t_{0}} \\
& \varepsilon^{1+1 / 2}: \quad \mathbf{x}_{2} \quad\left(d_{1}^{2} A \mathbf{u}_{3}+d_{2} A \mathbf{u}_{2}\right) \mathrm{e}^{\mathrm{i} \omega t_{0}} \\
& \varepsilon^{1+3 / 4}: \quad \mathbf{x}_{3} \quad\left(d_{1}^{3} A \mathbf{u}_{4}+2 d_{1} d_{2} A \mathbf{u}_{3}\right. \\
& \left.+d_{3} A \mathbf{u}_{2}\right) \mathrm{e}^{\mathrm{i} \omega t_{0}} \\
& \varepsilon^{2}: \quad \mathbf{x}_{4} \quad \hat{\mathbf{x}}_{4}+\left[3 d_{2} d_{2} A \mathbf{u}_{4}\right. \\
& \left.+\left(d_{2}^{2} A+2 d_{1} d_{3} A\right) \mathbf{u}_{3}+d_{4} A \mathbf{u}_{2}\right] \mathrm{e}^{\mathrm{i} \omega t_{0}} \\
& \varepsilon^{1+5 / 4}: \quad \mathbf{x}_{5} \quad \hat{\mathbf{x}}_{5}+\left[3\left(d_{1}^{2} d_{3} A+d_{1} d_{2}^{2} A\right) \mathbf{u}_{4}\right. \\
& +2\left(d_{2} d_{3} A+d_{1} d_{4} A\right) \mathbf{u}_{3} \\
& \left.+d_{5} A \mathbf{u}_{2}\right] \mathrm{e}^{\mathrm{i} \omega t_{0}} \\
& \varepsilon^{1+3 / 2}: \quad \mathbf{x}_{6} \quad \hat{\mathbf{x}}_{6}+\left(d_{2}^{3} A+3 d_{1}^{2} d_{4} A\right) \mathbf{u}_{4} \\
& +\left(d_{3}^{2} A+2 d_{2} d_{4} A+2 d_{1} d_{5} A\right) \mathbf{u}_{3} \\
& \left.+d_{6} A \mathbf{u}_{2}\right] \mathrm{e}^{\mathrm{i} \omega t_{0}}
\end{aligned}
$$




$$
\begin{array}{rll}
\varepsilon: & \lambda_{1}^{4} \quad \mathbf{v}_{4}^{\mathrm{H}} \mathbf{A}_{1} \mathbf{u}_{1} \\
\varepsilon^{5 / 4}: & 4 \lambda_{2} \lambda_{1}^{3} \quad f\left(\lambda_{1}\right) \\
\varepsilon^{3 / 2}: & 4 \lambda_{3} \lambda_{1}^{3} \quad \lambda_{1} f\left(\lambda_{1}, \lambda_{2}\right) \\
\varepsilon^{7 / 4}: & 4 \lambda_{4} \lambda_{1}^{3} \quad \lambda_{1} f\left(\lambda_{1}, \lambda_{2}, \lambda_{3}\right) \\
\varepsilon^{2}: & 4 \lambda_{5} \lambda_{1}^{3}+\lambda_{2}^{4} \quad \lambda_{1} f\left(\lambda_{1}, \lambda_{2}, \lambda_{3}, \lambda_{4}\right) \\
& +\mathbf{v}_{4}^{\mathrm{H}} \mathbf{A}_{1} \hat{\mathbf{w}}_{4} \\
& +\mathbf{v}_{4}^{\mathrm{H}} \mathbf{A}_{2} \mathbf{u}_{1}
\end{array}
$$

$$
\begin{array}{rll}
\varepsilon^{2}: & d_{1}^{4} A & 0 \\
\varepsilon^{1+5 / 4}: & 4 d_{2} d_{1}^{3} A & f\left(d_{1} A\right) \\
\varepsilon^{1+3 / 2}: & 4 d_{3} d_{1}^{3} A & d_{1} A f\left(d_{1} A, d_{2} A\right) \\
\varepsilon^{1+7 / 4}: & 4 d_{4} d_{1}^{3} A & d_{1} A f\left(d_{1} A, d_{2} A, d_{3} A\right) \\
\varepsilon^{3}: & 4 d_{5} d_{1}^{3} A+d_{2}^{4} A & d_{1} A f\left(d_{1} A, \ldots\right) \\
& +\mathbf{v}_{4}^{\mathrm{H}} \mathscr{F}_{\mathbf{x x}}^{0} \mathbf{u}_{1} \hat{\mathbf{x}}_{4} \\
& +\mathbf{v}_{4}^{\mathrm{H}} \mathbf{F}_{\mathbf{x x x}}^{0} \mathbf{u}_{1}^{3}
\end{array}
$$

$D^{4} A \quad \mathscr{L}\left(\boldsymbol{\mu} A, A^{2} \bar{A} ; \boldsymbol{\mu} \dot{A}, A \bar{A} \dot{A},+\quad\right)$
$\Delta \lambda^{4}+c_{1}(\boldsymbol{\mu}) \Delta \lambda^{3}+\quad+c_{4}(\boldsymbol{\mu}) \quad 0$
Finally, at order $\varepsilon^{2}$ (see Eqs. $\left(\mathrm{d}_{5}\right)$ ), a third order non trivial equation determines second order sensitivity $\lambda_{2}$, if $\mathbf{v}_{4}^{\mathrm{H}} \mathbf{A}_{2} \mathbf{u}_{1} \neq 0$. Therefore $\lambda \quad \lambda_{0} \quad O\left(\varepsilon^{1 / 2}\right)$. It is possible to check that $\mathbf{w}_{1}, \mathbf{w}_{3}, \ldots$ also vanish. One can therefore adopt a series expansion of $\varepsilon^{2 / m}$, both for the eigenvalue $\lambda$ and for the eigenvector $\mathbf{w}$, furnishing non trivial infor mation at each step.

The bifurcation problem is now addressed. By using expansions $\left(\mathrm{a}^{\prime}\right)$ in Table 1 the perturbation equations $\left(b^{\prime}\right)$ follows. The analogy with Eqs. (b) should be noted. At the $\varepsilon^{2}$ order the perturbation $\mathbf{F}_{\mathbf{x x}}^{0} \mathbf{x}_{0}^{2}$ appears. How ever, since $\mathbf{x}_{0}$ is a harmonic function of time, this term is not resonant, since it is the sum of a double harmonic and a constant term. There therefore occurs a circum stance similar to the singular perturbation of the sensi tivity problem. In contrast, resonance is produced by the cubic nonlinearity $\mathbf{F}_{\mathbf{x x \mathbf { x }}}^{0} \mathbf{x}_{0}^{3}$, appearing at the $\varepsilon^{3}$ order. As a consequence, odd terms in the expansions of $\mathbf{x}$ and $D$ vanish. This suggests omitting them in advance, in or der to obtain simpler equations. Moreover, the scaling $\boldsymbol{\mu}=\varepsilon^{2} \hat{\boldsymbol{\mu}}$ must be introduced to render $\mathbf{F}_{\mathbf{x} \boldsymbol{\mu}}^{0} \mathbf{x}_{0} \hat{\boldsymbol{\mu}}$ of the same order as the resonant term $\mathbf{F}_{\mathbf{x x x}}^{0} \mathbf{x}_{0}^{3}$. The algorithm of Sec tion 3.1 follows.

\section{Odd $m \quad 3$ case}

This case is illustrated in Table 2. In sensitivity anal ysis, similarly to the previous case, when the singular perturbation $\mathbf{v}_{3}^{\mathrm{H}} \mathbf{A}_{1} \mathbf{u}_{1}=0$ occurs, the odd terms of the $\lambda$ series all vanish. However, while odd terms $\mathbf{w}_{1}$ $\mathbf{w}_{3} 0$, in contrast $\mathbf{w}_{5}, \mathbf{w}_{7}, \ldots$, are different from zero, so that a complete series of $\varepsilon^{1 / m}$ must be used for odd $m$. However, an expansion of $\mathbf{w}$ based on fractional pow ers of $\varepsilon^{2 / m}$, would not produce an $\varepsilon^{2}$ order perturbation equation in which to place the perturbation $\mathbf{A}_{2} \mathbf{w}_{0}$. A similar problem arises in the bifurcation analysis, where an even series would make it impossible to place $\mathbf{F}_{\mathbf{x x}}^{0} \mathbf{x}_{0}^{2}$. Therefore the algorithm of Section 3.2 must be used.

\section{Appendix B. Particular solutions and coefficients}

The vectors and matrices $\mathbf{z}_{i j}, \mathbf{Z}_{i \boldsymbol{\mu}}$ appearing in Eqs. (17) are obtained by solving the following linear alge braic problems:

$\left(\mathrm{i} 2 \omega \mathbf{E} \quad \mathbf{F}_{\mathbf{x}}^{0}\right) \mathbf{z}_{11}=\mathbf{F}_{\mathbf{x x}}^{0} \mathbf{u}_{1}^{2}$

$\left(\begin{array}{ll}\mathrm{i} 2 \omega \mathbf{E} & \mathbf{F}_{\mathbf{x}}^{0}\end{array}\right) \mathbf{z}_{12}=\mathbf{z}_{11}+\mathbf{F}_{\mathbf{x x}}^{0} \mathbf{u}_{1} \mathbf{u}_{2}$

$\left(\mathbf{F}_{\mathbf{x}}^{0}\right) \mathbf{z}_{11}=\mathbf{F}_{\mathbf{x x}}^{0} \mathbf{u}_{1} \mathbf{u}_{1}$

$\left(\mathbf{F}_{\mathbf{x}}^{0}\right) \mathbf{z}_{12}=\frac{1}{2}\left[\begin{array}{ll}\mathbf{z}_{11} & \mathbf{z}_{11}+2 \mathbf{F}_{\mathbf{x x}}^{0} \mathbf{u}_{1} \mathbf{u}_{2}\end{array}\right]$

$\left(\mathrm{i} 3 \omega \mathbf{E} \quad \mathbf{F}_{\mathbf{x}}^{0}\right) \mathbf{z}_{111}=\frac{1}{2}\left[\mathbf{F}_{\mathbf{x x}}^{0} \mathbf{z}_{11} \mathbf{u}_{1}+\frac{1}{3} \mathbf{F}_{\mathbf{x x x}}^{0} \mathbf{u}_{1}^{3}\right]$

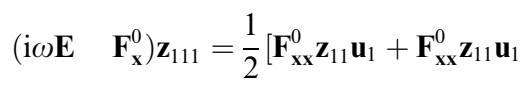

$$
\begin{gathered}
+\mathbf{F}_{\mathbf{x x}}^{0} \mathbf{z}_{11} \mathbf{u}_{1}+\mathbf{F}_{\mathbf{x x x}}^{0} \mathbf{z}_{11} \mathbf{u}_{1}^{2} \mathbf{u}_{1} \\
\mathbf{v}_{2}^{\mathrm{T}}\left(\mathbf{F}_{\mathbf{x x}}^{0} \mathbf{z}_{11} \mathbf{u}_{1}+\mathbf{F}_{\mathbf{x x}}^{0} \mathbf{z}_{11} \mathbf{u}_{1}\right. \\
\left.\left.+\mathbf{F}_{\mathbf{x x}}^{0} \mathbf{z}_{11} \mathbf{u}_{1}+\mathbf{F}_{\mathbf{x x x}}^{0} \mathbf{z}_{11} \mathbf{u}_{1}^{2} \mathbf{u}_{1}\right) \mathbf{u}_{2}\right]
\end{gathered}
$$

$\left(\mathrm{i} \omega \mathbf{E} \quad \mathbf{F}_{\mathbf{x}}^{0}\right) \mathbf{Z}_{1 \mu} \hat{\boldsymbol{\mu}}=\mathbf{F}_{\mathbf{x} \gamma}^{0} \mathbf{u}_{1} \hat{\boldsymbol{\mu}} \quad \mathbf{v}_{2}^{\mathrm{T}}\left(\mathbf{F}_{\mathbf{x} \gamma}^{0} \mathbf{u}_{1} \hat{\boldsymbol{\mu}}\right) \mathbf{u}_{2}$

where $\mathbf{E}$ is the identity matrix. Since $\left(i \omega \mathbf{E} \quad \mathbf{F}_{\mathbf{x}}{ }^{0}\right)$ is singular, the solutions of Eqs. (B.1 $1_{5,6}$ ) are not unique. To avoid indeterminacies, a normalization condition 
Table 2

Eigenpair sensitivity and bifurcation analysis: $m=3$

$\mathbf{w} \quad \mathbf{w}_{0}+\varepsilon^{1 / 3} \mathbf{w}_{1}+\varepsilon^{2 / 3} \mathbf{w}_{2}+$

$\lambda \quad \lambda_{0}+\varepsilon^{1 / 3} \lambda_{1}+\varepsilon^{2 / 3} \lambda_{2}+$

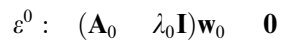

$$
\begin{aligned}
& \varepsilon^{1 / 3}:\left(\begin{array}{lll}
\mathbf{A}_{0} & \left.\lambda_{0} \mathbf{I}\right) \mathbf{w}_{1} & \lambda_{1} \mathbf{w}_{0}
\end{array}\right. \\
& \varepsilon^{2 / 3}:\left(\begin{array}{lll}
\mathbf{A}_{0} & \left.\lambda_{0} \mathbf{I}\right) \mathbf{w}_{2} & \lambda_{1} \mathbf{w}_{1}+\lambda_{2} \mathbf{w}_{0}
\end{array}\right. \\
& \varepsilon: \quad\left(\begin{array}{lll}
\mathbf{A}_{0} & \lambda_{0} \mathbf{I}
\end{array}\right) \mathbf{w}_{3} \quad \lambda_{1} \mathbf{w}_{2}+\lambda_{2} \mathbf{w}_{1} \\
& +\lambda_{3} \mathbf{w}_{0} \quad \mathbf{A}_{1} \mathbf{w}_{0}
\end{aligned}
$$

$\varepsilon^{4 / 3}:\left(\begin{array}{lll}\mathbf{A}_{0} & \lambda_{0} \mathbf{I}\end{array}\right) \mathbf{w}_{4} \quad \lambda_{1} \mathbf{w}_{3}+\lambda_{2} \mathbf{w}_{2}$

$$
+\lambda_{3} \mathbf{w}_{1}+\lambda_{4} \mathbf{w}_{0} \quad \mathbf{A}_{1} \mathbf{w}_{1}
$$

$\varepsilon^{5 / 3}: \quad\left(\begin{array}{lll}\mathbf{A}_{0} & \left.\lambda_{0} \mathbf{I}\right) \mathbf{w}_{5} & \lambda_{1} \mathbf{w}_{4}+\lambda_{2} \mathbf{w}_{3}\end{array}\right.$

$$
+\quad+\lambda_{5} \mathbf{w}_{0} \quad \mathbf{A}_{1} \mathbf{w}_{2}
$$

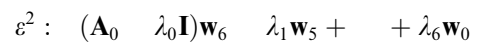
$\mathbf{A}_{1} \mathbf{w}_{3}$

$\mathbf{A}_{2} \mathbf{w}_{0}$

$$
\begin{array}{rll}
\varepsilon^{0}: & \mathbf{w}_{0} & \mathbf{u}_{1} \\
\varepsilon^{1 / 3}: & \mathbf{w}_{1} & \lambda_{1} \mathbf{u}_{2} \\
\varepsilon^{2 / 3}: & \mathbf{w}_{2} & \lambda_{1}^{2} \mathbf{u}_{3}+\lambda_{2} \mathbf{u}_{2} \\
\varepsilon: & \mathbf{w}_{3} & \hat{\mathbf{w}}_{3}+2 \lambda_{1} \lambda_{2} \mathbf{u}_{3}+\lambda_{3} \mathbf{u}_{2} \\
\varepsilon^{4 / 3}: & \mathbf{w}_{4} & \hat{\mathbf{w}}_{4}+\left(\lambda_{2}^{2}+2 \lambda_{1} \lambda_{3}\right) \mathbf{u}_{3} \\
& +\lambda_{4} \mathbf{u}_{2}
\end{array}
$$

$$
\Delta \lambda^{3}+c_{1}(\boldsymbol{\mu}) \Delta \lambda^{2}+\quad+c_{3}(\boldsymbol{\mu}) \quad 0
$$

$\begin{aligned} \mathbf{x} & \varepsilon\left(\mathbf{x}_{0}+\varepsilon^{1 / 3} \mathbf{x}_{1}+\varepsilon^{2 / 3} \mathbf{x}_{2}+\quad\right) \\ \mathrm{d} / \mathrm{d} t & d_{0}+\varepsilon^{1 / 3} d_{1}+\varepsilon^{2 / 3} d_{2}+\end{aligned}$

$$
\begin{aligned}
& \varepsilon: \quad\left(\begin{array}{ll}
d_{0} & \left.\mathbf{F}_{\mathbf{x}}^{0}\right) \mathbf{x}_{0} \quad 0
\end{array}\right. \\
& \varepsilon^{1+1 / 3}:\left(\begin{array}{lll}
d_{0} & \left.\mathbf{F}_{\mathbf{x}}^{0}\right) \mathbf{x}_{1} \quad d_{1} \mathbf{x}_{0}
\end{array}\right. \\
& \varepsilon^{1+2 / 3}:\left(\begin{array}{llll}
d_{0} & \left.\mathbf{F}_{\mathbf{x}}^{0}\right) \mathbf{x}_{2} & d_{1} \mathbf{x}_{1} & d_{2} \mathbf{x}_{0}
\end{array}\right. \\
& \varepsilon^{2}: \quad\left(\begin{array}{llll}
d_{0} & \left.\mathbf{F}_{\mathbf{x}}^{0}\right) \mathbf{x}_{3} & d_{1} \mathbf{x}_{2} & d_{2} \mathbf{x}_{1}
\end{array}\right. \\
& d_{3} \mathbf{x}_{0}+1 / 2 \mathbf{F}_{\mathbf{x x}}^{0} \mathbf{x}_{0}^{2} \\
& \varepsilon^{1+4 / 3}:\left(\begin{array}{llll}
d_{0} & \left.\mathbf{F}_{\mathbf{x}}^{0}\right) \mathbf{x}_{4} & d_{1} \mathbf{x}_{3} & d_{2} \mathbf{x}_{2}
\end{array}\right. \\
& d_{3} \mathbf{x}_{1} \quad d_{4} \mathbf{x}_{0}+\mathbf{F}_{\mathbf{x x}}^{0} \mathbf{e} 2 \mathbf{x}_{0} \mathbf{x}_{1} \\
& \varepsilon^{1+5 / 3}: \quad\left(\begin{array}{llll}
d_{0} & \left.\mathbf{F}_{\mathbf{x}}^{0}\right) \mathbf{x}_{5} & d_{1} \mathbf{x}_{4} & d_{2} \mathbf{x}_{3}
\end{array}\right. \\
& +\quad d_{5} \mathbf{x}_{0}+\mathbf{F}_{\mathbf{x x}}^{0} \mathbf{x}_{0} \mathbf{x}_{2}+\mathbf{F}_{\mathbf{x x}}^{0} \mathbf{x}_{1}^{2} \\
& \varepsilon^{3}: \quad\left(\begin{array}{llll}
d_{0} & \left.\mathbf{F}_{\mathbf{x}}^{0}\right) \mathbf{x}_{6} & d_{1} \mathbf{x}_{5}+\quad d_{6} \mathbf{x}_{0}
\end{array}\right. \\
& +\mathbf{F}_{\mathbf{x x}}^{0}\left(\mathbf{x}_{0} \mathbf{x}_{3}+\mathbf{x}_{1} \mathbf{x}_{2}+\right) \\
& +1 / 6 \mathbf{F}_{\mathbf{x x x}}^{0} \mathbf{x}_{0}^{3}+\mathbf{F}_{\mathbf{x} \boldsymbol{\mu}}^{0} \mathbf{x}_{0} \hat{\boldsymbol{\mu}}
\end{aligned}
$$

$$
\begin{array}{rll}
\varepsilon: & \mathbf{x}_{0} & A \mathbf{u}_{1} e^{i \omega t 0} \\
\varepsilon^{1+1 / 3}: & \mathbf{x}_{1} & d_{1} A \mathbf{u}_{2} e^{i \omega t 0} \\
\varepsilon^{1+2 / 3}: & \mathbf{x}_{2} & \left(d_{1}^{2} A \mathbf{u}_{3}+d_{2} A \mathbf{u}_{2}\right) \mathrm{e}^{\mathrm{i} \omega t 0} \\
\varepsilon^{2}: & \mathbf{x}_{3} & \hat{\mathbf{x}}_{3}+\left[2 d_{1} d_{2} A \mathbf{u}_{3}+d_{3} A \mathbf{u}_{2}\right] \mathrm{e}^{\mathrm{i} \omega t 0} \\
\varepsilon^{1+4 / 3}: & \mathbf{x}_{4} & \hat{\mathbf{x}}_{4}+\left[\left(d_{2}^{2} A+2 d_{1} d_{3} A\right) \mathbf{u}_{3}\right. \\
& & \left.+d_{4} A \mathbf{u}_{2}\right] e^{i \omega t 0}
\end{array}
$$

$$
\begin{array}{rll}
\varepsilon^{2}: & d_{1}^{3} A & 0 \\
\varepsilon^{1+4 / 3}: & 3 d_{2} d_{1}^{2} A & f\left(d_{1} A\right) \\
\varepsilon^{1+5 / 3}: & 3 d_{3} d_{1}^{2} A & d_{1} A f\left(d_{1} A, d_{2} A\right) \\
\varepsilon^{3}: & 3 d_{4} d_{1}^{2} A+d_{2}^{3} A \quad d_{1} A f\left(d_{1} A, \ldots\right) \\
& +\mathbf{v}_{3}^{\mathrm{H}} \mathbf{F}_{\mathbf{x x}}^{0} \mathbf{u}_{1} \hat{\mathbf{x}}_{3} \\
& +\mathbf{v}_{4}^{\mathrm{H}} \mathbf{F}_{\mathbf{x x x}}^{0} \mathbf{u}_{1}^{3}+\ldots
\end{array}
$$

$D^{3} A \quad \mathscr{L}\left(\boldsymbol{\mu} A, A^{2} \bar{A} ; \boldsymbol{\mu} \dot{A}, A \bar{A} \dot{A},+\quad\right)$ must be enforced, e.g. by imposing $\mathbf{v}_{1}^{\mathrm{H}} \mathbf{z}_{111}=0$, $\mathbf{v}_{1}^{\mathrm{H}} \mathbf{Z}_{1 \boldsymbol{\mu}}=\mathbf{0}$.
The coefficients $C_{i j h}$ and the row vectors $\mathbf{C}_{i \boldsymbol{\mu}}$ appear ing in Eqs. (20) are given by: 


$$
\begin{aligned}
& \mathbf{C}_{1 \mu} \hat{\mu}= \mathbf{v}_{2}^{\mathrm{T}} \mathbf{F}_{\mathbf{x} \gamma}^{0} \mathbf{u}_{1} \hat{\boldsymbol{\mu}} \\
& \mathbf{C}_{2 \mu} \hat{\boldsymbol{\mu}}= \mathbf{v}_{2}^{\mathrm{T}}\left(-\mathbf{Z}_{1 \mu} \hat{\boldsymbol{\mu}}+\mathbf{F}_{\mathbf{x \mu}}^{0} \mathbf{u}_{2} \hat{\boldsymbol{\mu}}\right) \\
& C_{11 \overline{1}}=1 / 2 \mathbf{v}_{2}^{\mathrm{T}}\left(\mathbf{F}_{\mathbf{x x}}^{0} \mathbf{z}_{1 \overline{1}} \mathbf{u}_{1}+\mathbf{F}_{\mathbf{x x}}^{0} \mathbf{z}_{11} \overline{\mathbf{u}}_{1}+\mathbf{F}_{\mathbf{x x}}^{0} \overline{\mathbf{z}}_{1 \overline{1}} \mathbf{u}_{1}\right. \\
&\left.\quad+\mathbf{F}_{\mathbf{x x x}}^{0} \mathbf{u}_{1}^{2} \overline{\mathbf{u}}_{1}\right) \\
& C_{1 \overline{1} 2}=\mathbf{v}_{2}^{\mathrm{T}}\left(-2 \mathbf{z}_{11 \overline{1}}+\mathbf{F}_{\mathbf{x x}}^{0} \mathbf{z}_{\overline{1} \overline{2}} \mathbf{u}_{1}+\mathbf{F}_{\mathbf{x x}}^{0} \mathbf{z}_{12} \overline{\mathbf{u}}_{1}\right. \\
& \quad+1 / 2 \mathbf{F}_{\mathbf{x x}}^{0} \mathbf{z}_{1 \overline{1}} \mathbf{u}_{2}+1 / 2 \mathbf{F}_{\mathbf{x x}}^{0} \overline{\mathbf{z}}_{1 \overline{1}} \mathbf{u}_{2} \\
&\left.\quad+\mathbf{F}_{\mathbf{x x x}}^{0} \mathbf{u}_{1} \overline{\mathbf{u}}_{1} \mathbf{u}_{2}\right) \\
& C_{11 \overline{2}}=\mathbf{v}_{2}^{\mathrm{T}}\left(-\mathbf{z}_{11 \overline{1}}+\mathbf{F}_{\mathbf{x x}}^{0} \overline{\mathbf{z}}_{\overline{1} 2} \mathbf{u}_{1}+1 / 2 \mathbf{F}_{\mathbf{x x}}^{0} \mathbf{z}_{11} \overline{\mathbf{u}}_{2}\right. \\
&\left.\quad+1 / 2 \mathbf{F}_{\mathbf{x x x}}^{0} \mathbf{u}_{1}^{2} \overline{\mathbf{u}}_{2}\right)
\end{aligned}
$$

\section{References}

[1] Guckenheimer J, Holmes P. Nonlinear oscillations, dynamical systems, and bifurcations of vector fields. New York: Springer-Verlag; 1983.

[2] Arnold VI. Geometrical methods in the theory of ordinary differential equations. New York: Springer Verlag; 1982. [Russian original, Moscow 1977].

[3] Nayfeh AH. Introduction to perturbation techniques. New York: Wiley-Interscience; 1991.

[4] Luongo A, Paolone A. Perturbation methods for bifurcation analysis from multiple nonresonant complex eigenvalues. Nonlinear Dynam 1997;14:193-210.

[5] Luongo A, Paolone A, Di Egidio A. Multiple time scales analysis for 1:2 and 1:3 resonant Hopf bifurcations. Nonlinear Dynam 2003;34(3-4):269-81.

[6] Luongo A, Paolone A. Multiple scale analysis for divergence-Hopf bifurcation of imperfect symmetric systems. J Sound Vibrat 1998;218:527-39.

[7] Luongo A, Di Egidio A, Paolone A. Multiple time scale analysis for bifurcation from a multiple-zero eigenvalue. AIAA J 2003;41(6):1143-50.

[8] Luongo A, Di Egidio A, Paolone A. Multiple scale bifurcation analysis for finite-dimensional autonomous systems. Recent Research Developments in Sound and Vibration; Kerala (India). Transworld Res Network 2002;1:161-201.
[9] Cohen DS. Bifurcation from multiple complex eigenvalues. J Math Anal Appl 1977;57:505-21.

[10] Wen GL. Codimension-2 Hopf bifurcation of a twodegree-of-freedom vibro-impact system. J Sound Vibrat 2000;242(3):475-85.

[11] Das SL, Chatterjee A. Multiple scales without center manifold reductions for delay differential equations near Hopf bifurcations. Nonlinear Dynam 2002;30:323-35.

[12] Yu P. Analysis on double Hopf bifurcation using computer algebra with the aid of multiple scales. Nonlinear Dynam 2002;27:19-53.

[13] Natsiavas S. Free vibration of two coupled nonlinear oscillators. Nonlinear Dynam 1994;6:69-86.

[14] Natsiavas S. Free vibration in a class of self-excited oscillators with 1:3 internal resonance. Nonlinear Dynam 1997;12:109-28.

[15] Gils SA, Krupa MPM, Langford WF. Hopf bifurcation with nonsemisimple 1:1 resonance. Nonlinearity 1990; $3: 1-26$.

[16] Gattulli V, Di Fabio F, Luongo A. One to one resonant double Hopf bifurcation in aeroelastic osciilators with tuned mass dampers. J Sound Vibrat 2003;262:201-17.

[17] Luongo, A., Di Egidio A., Bifurcation equations through multiple-scales analysis for a continuous model of a planar beam. Nonlinear Dynam, 2004 (in press).

[18] Troger H. Methods for dimension reduction and their application in nonlinear dynamics. Int J Solids Struct 2001;38:2131-47.

[19] Luongo A, Paolone A. On the reconstitution problem in the multiple time scale method. Nonlinear Dynam 1999;14:133-56.

[20] Nayfeh AH. Topical course on nonlinear dynamics, SocietàItaliana di Fisica, Santa Margherita di Pula, Sardinia, Perturbation Methods Nonlinear Dynam 1985.

[21] Luongo A. Eigensolutions sensitivity for nonsymmetric matrices with repeated eigenvalues. AIAA J 1993;31: 1321-1328.

[22] Luongo A, Di Egidio A, Paolone A. On the proper form of the amplitude modulation equations for resonant systems. Nonlinear Dynam 2002;27:237-54.

[23] Piccardo G. A methodology for the study of coupled aeroelastic phenomena. J Wind Eng Ind Aerodynam 1993;48:241-52. 